\title{
Numerical Simulations of a Quiet SuperSonic Technology (QueSST) Aircraft Preliminary Design
}

\author{
David J. Friedlander ${ }^{1}$, ChristopherM. Heath ${ }^{2}$, and Raymond S. Castner ${ }^{3}$ \\ NASA Glenn Research Center, Cleveland, $\mathrm{OH}, 44135$
}

\begin{abstract}
Reynolds Averaged Navier-Stokes (RANS) simulations were performed on a Lockheed Martin Quiet SuperSonic Technology (QueSST) aircraft preliminary design to as s ess inlet performance. The FUN3D flow solver and its adjoint-based grid refinement capability were usedfor the simulations in hopes of determining internal "best practices" for predicting inlet performance on top-aft-mounted inlets. Several parameters were explored including tetrahedral us. pentahedral cells in/around the boundary-layer regions, an engine axisaligned linear pressure sensor us. a pressure box objective as the gridadaptation metric, and the number of grid adaptation cycles performed. Additional simulations were performed on manually refined grids for comparison with the adjoint-based adapted grids. Results showed poor agreement in predicted inlet performance on the refined grids compared to experimental data. This was true regardless of whether the refinement was adjoint-bas ed or manual, the cell type in/near the boundary-layer regions, or the grid adaptation metric used. In addition, the 40-probe total pressure recovery was shown to decrease asymptotically as the number of adaptation cycles is increased. Solutions on the unadapted grids generally had better agreement with experimental data than their refinedgrid counterparts.
\end{abstract}

\section{Nomenclature}

$\begin{array}{ll}D P C P & =\text { inlet circumferential distortion } \\ D P R P & =\text { inlet radial distortion } \\ M & =\text { Mach number } \\ m_{2} / m_{0} & =\text { inlet mass flow rate ratio } \\ p, p_{t} & =\text { static and total pres sure } \\ p_{t, 2} / p_{t, \infty} & =\text { inlet total pressure recovery } \\ u & =\text { streamwise velocity } \\ x, y, z & =\text { cartesian coordinates } \\ y^{+} & =\text {non-dimensional wall distance } \\ \alpha & =\text { angle of attack } \\ \beta & =\text { sides lip angle } \\ \sigma & =\text { standard deviation } \\ \infty & =\text { freestream }\end{array}$

\section{Introduction}

$\Lambda$ LTHOUGH there has not been a commercial supersonic flight since the retirement of the Concorde fleet in 2003, the aviation community has shown interest in bringing back commercial supersonic tran sports, with several companies already pursuing efforts ${ }^{1-3}$. These efforts are on-going despite the current ban on supersonic flight over U.S. territories due to the loudness of the sonic booms produced by aircraft flying at supersonic speeds ${ }^{4}$. NASA has taken an interest in paving the way for commercial supersonic transport aircraft in the United States ${ }^{5}$ and has gone as far as devoting one of the x-planes in the New Aviation Horizon s in it iative to demonstrating that an aircraft flying supersonically can generate a quiet sonic boom ${ }^{6}$. This low boom flight demonstrator aircraft was contracted out to Lockheed Martin by NASA to develop the aircraft through the

\footnotetext{
${ }^{1}$ Aerospace Engineer, Inlets and Nozzles Branch, d.j.friedlander@nasa.gov, AIAA Member

${ }^{2}$ Aerospace Engineer, Propulsion Systems Analysis Branch, chris topher.m.heath@nasa.gov, Non-Member

${ }^{3}$ Aeros pace Engineer, Inlets and Nozzles Branch, raymond.s.castner@ nasa.gov, AIAA Associate Fellow
}

1

American Institute of Aeronautics and Astronautics 
preliminary design review ${ }^{7}$. While several incarnations of the aircraft were analyzed by both Lockheed Martin engineers and NASA researchers, the C607.1 version of the Quiet SuperSonic Technology (QueSST) a ircraft was chosen for wind tunnel aerodynamic and propulsion tests in the NASA Glenn Res earch Center's (GRC's) 8'x6' Supersonic Wind Tunnel(SWT) during the first half of 2017. This paperfocuses on the Reynolds Averaged NavierStokes (RANS) computational fluid dynamic (CFD) simulations performed by the NASA GRC researchers that supported the propulsion wind tunnel test effort.

\section{Geometry and Numerical Modeling}

\section{A. QueSST C607.1 Geometry}

The QueSST aircraft preliminary design, shown in Fig. 1, is a single engine aircraft, with the engine topmounted at the aft-end of the fuselage. The in let is an external compres sion diverterless bump inlet that compresses the flow external of the inlet duct while diverting the boundary-layer flow away from the engine intake. The C607.1 version, shown in Fig. 2 with features highlighted in Fig. 3, had modified internal inlet contours with resp ect to its predecessors in order to improve inlet performance. The inlet itself had a throat area of 510in ${ }^{2}$ and a subsonic diffuser length of 96in. Vortex generators were situated approximately 10 inlet diameters upstream of the in let in order to help mitigate boundary-layer flow from being ingested by the inlet. Approximately 15 inlet diameters upstream of the vortex generators is a camera fairing for housing an external camera system. The camera system is required to help aid the pilot with take-offs and landings due to the minimal visibility from the cockpit. The presented simulations used a 9.5\% scaled version of the C607.1 aircraft, which is consistent with the scale of the wind tunnel model used in the 8'x6' SWT aerodynamic and propulsion tests.

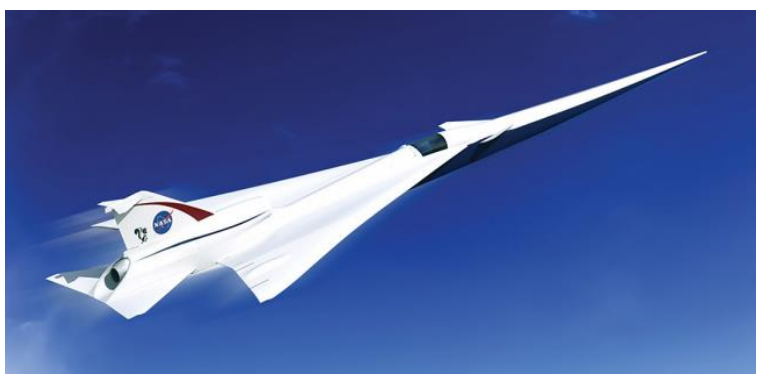

Figure 1. Artist's concept of the Lock heed Martin QueSS Taircraft preliminary design ${ }^{5}$.

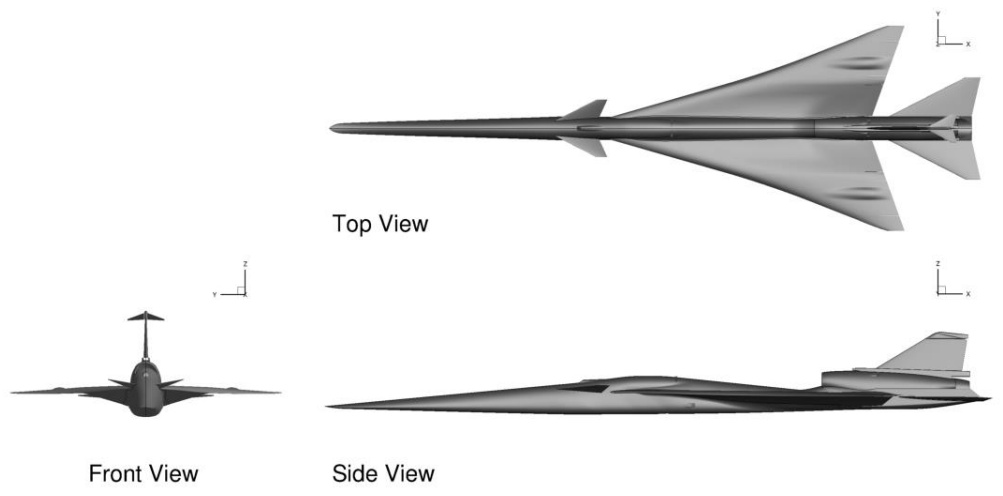

Figure 2. QueSST C607.1 aircraft preliminary design. 


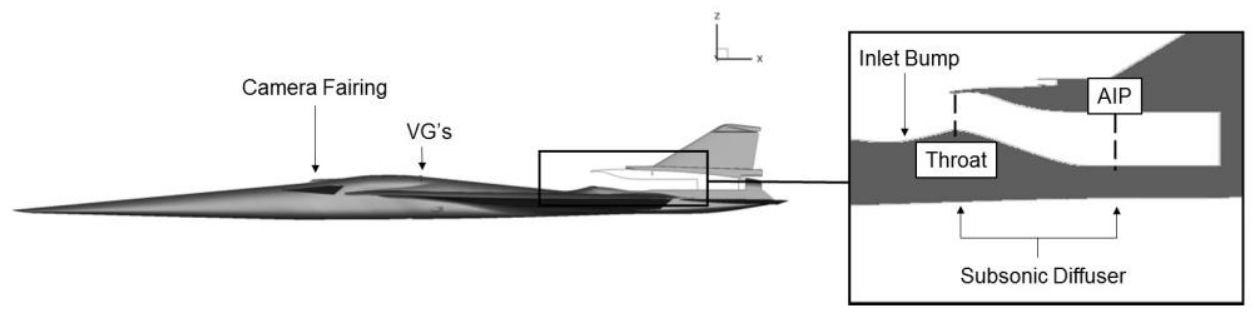

Figure 3. QueSST C607.1 aircraft features, including the aerodynamic interface plane (AIP).

\section{B. Flow Solver}

NASA's FUN3D ${ }^{8}$ code was used for all simulations. FUN3D is a node-based production level codedeveloped and maintained at the NASA Langley Research Center. It can solve 2D/3D Euler and RANS equations for incompressible and compressible flows on unstructured grids. The FUN3D flow solver was ch osen for two main reasons: 1) it can handle complex geometries represented by unstructured grids and 2) it has the capability to performadjoint-based grid adaptation. The adjoint-based grid adaption uses the refine/one library ${ }^{9}$, which require s "freezing" all boundary-layer (BL) cells within a user-specified distance from no-slip walls. FUN3D's adjointbased grid adaptation capability has been used exten sively with external flow applications including complexnozzle plumes $^{9-11}$, sonic boom predictions ${ }^{12}$, and internal flow applications such as s-ducts ${ }^{13}$. The adjoint-based grid adaptation works by reducing the grid spatial discretization error with respect to a specified flow field metric by leveraging flow solution sensitivities. For the presented simulations, the pressure within the inlet duct was chosen as the flow field metric for adaptation.

\section{Grid Adaptation Parameters}

In order to develop in ternal "best practices" for capturing top-aft-mounted inlet performance, three parameters were chosen to explore their sensitivities to the predicted in let performance. These included the cell type in/near the boundary-layer, the grid adaptation metric, and the number of grid adaptation cycles. The celltype looked at u sing tetrahedrals or pentahedrals (i.e. pris ms and pyramids) in and around the boundary-layer regions. The two grid adaptation metrics that were explored were an engine axis-aligned linear pressure sensor and a pressure box objective. The linear pressure sensor objective works by minimizing the discretization error around a linear pressure "sensor" while the pressure box objective works by computing the RMS values of pressure. Each objective is only activate within a user specified region of the flow field domain. Finally, the number of grid adaptation cy cles was varied from 8 to 16 cycles. Table 1 summarizes the combinations of the parameters that were simulated.

Table 1: Grid adaptation cases.

\begin{tabular}{|c|c|c|c|}
\hline Case \# & BL Cell Type & Adaptation Metric & Adaptation Cycles \\
\hline 1 & Tetrahedral & NA & 0 \\
\hline 2 & Tetrahedral & Linear Pres sure Sensor & 8 \\
\hline 3 & Pentahedral & NA & 0 \\
\hline 4 & Pentahedral & Pressure Box & 8 \\
\hline 5 & Tetrahedral & PressureBox & $8^{*}$ \\
\hline 6 & Tetrahedral & PressureBox & $16^{*}$ \\
\hline 7 & Pentahedral & Pressure Box & $8^{*}$ \\
\hline 8 & Pentahedral & PressureBox & $16^{*}$ \\
\hline
\end{tabular}

*reduced number of additionalnodes/adaptation cycle.

\section{Initial and Manually RefinedGrids}

An unstructured surface grid was generated using the Pointwise ${ }^{14}$ grid generation software while three different initial volume grids were generated using the AFLR $3{ }^{15}$ code. AFLR3 is a research code developed at Mississippi State University that generates unstructured tetrahedral/pentahedral volume grids via the Advancing -Front/Local Reconstruction method ${ }^{16,17}$. The three initial volume grids were differentiated as follows; grid \#1 consisted of all tetrahedral cells (referred to as the tetrahedral boundary-layer grid), grid \#2 contained a mix of tetrahedral and pentahedral cells (referred to as the pentahedral boundary-layergrid), and grid \#3 was a smoothed vers ion of grid 
\#2 (referred to as the pentahedral boundary-layer smooth grid). Viscous spacing was such that the $y^{+}$value was less than 0.2. Nodal count for all initial grids was 33.4 million. Due to symmetry, only half of the aircraft was modeled.

In addition, two manually refined grids, one based off of grid \#1 and the other based off of grid \#2, were developed following the same process as the initial grids for comparison with the adapted grids. Refine ment to ok the form of uniformly increasing the nodal count on the surface grid connectors by a factor of 1.5 and decreasing the initial spacing off the viscous surfaces by a factor of 1.5. This resulted in volume grids with 92.3 million node s for the tetrahedral boundary-layer based grid and 91.8 million nodes for the pentahedral boundary-layer based grid.

\section{E. Flow Conditions}

Three different experimental set points were chosen for the simulations, with details outlined in Table 2. Most of the simulations focused on the experimental data points condition referred to as Reading 1755, which at $M_{\infty}=1.46$ was slightly higher than the aircraft's designed freestream cruise condition of $M_{\infty}=1.42$. Additionally, a lower supersonic point of $M_{\infty}=1.35$ (Reading 1771) and a low subsonic point of $M_{\infty}=0.30$ (Reading 2033) were chosen for additional comparis ons.

Table 2: Set point conditions.

\begin{tabular}{|c|c|c|c|}
\hline Reading \# & Mach Number & $\alpha(\mathrm{deg})$ & $\beta(\mathrm{deg})$ \\
\hline 1755 & 1.46 & 2.0 & 0.0 \\
\hline 1771 & 1.35 & 3.0 & 0.0 \\
\hline 2033 & 0.30 & 3.0 & 0.0 \\
\hline
\end{tabular}

\section{F. Boundary Conditions, Initial Solutions, and Turbulence Modeling}

A combination of freestream and farfield boundary conditions were applied to the outer boundaries of the computational domain, shown in Fig. 4 for the supersonic flow cases and Fig. 5 for the subsonic flow case. An extrapolation boundary condition was applied to the outflow boundary for the supersonic flow cases while a farfield boundary condition was applied for the subsonic flow case. Mass flow through the in let was set indirectly by specifying the inlet exit plane Mach number, which in turn set the back pressure within the inlet duct. Setting the mass flow through the inlet in this manor was in lieu of modeling the mass flow plug that was used in the experiment. Flow through the nozzle was set by imposing a subsonic inflow boundary condition at the nozzle inflow plane, where the total pressure and total temperature ratios were specified. Initial solutions were set to uniformflow at the freestream conditions with the exception of theinlet duct, which was initialized at a subsonic uniformflow. All simulations used the Spalart-Allmaras turbulence model ${ }^{18}$.

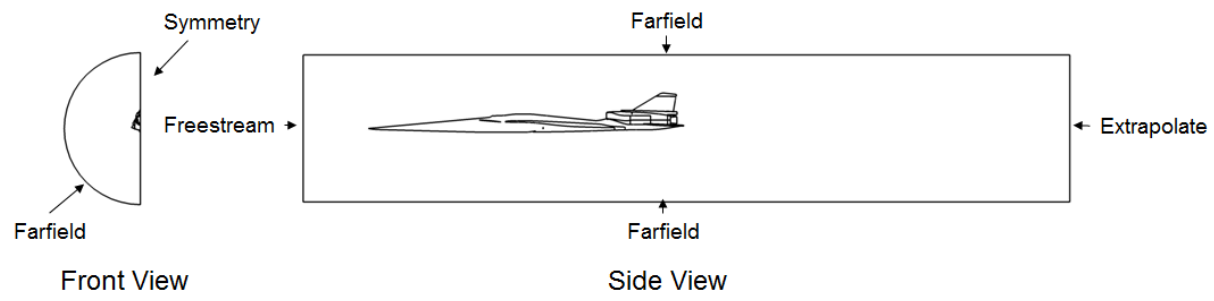

Figure 4. Boundary conditions for supers onic flow conditions.

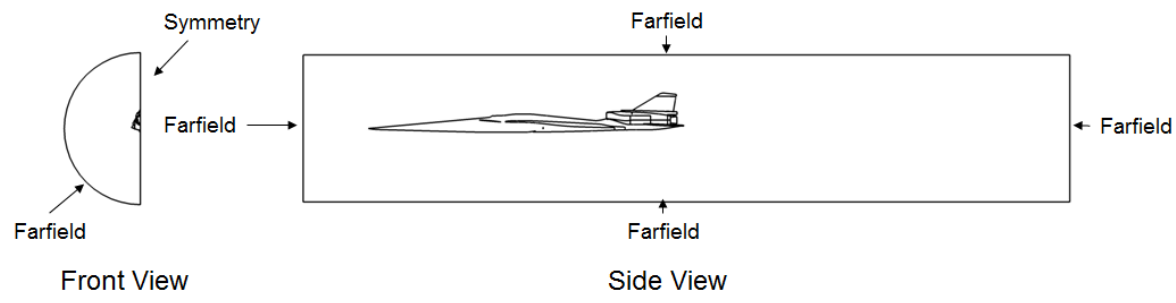

Figure 5. Boundary conditions for subs onic flow condition. 


\section{Results}

The presented results will mostly focus on the solutions along the aircraft centerline. While the in let was the main area of interest, two additional stations upstreamof the in let were chosen for comparis on with experimental data: the area around the camera faring and the area at theinlet bump. Figure 6 shows all of the areas of intere st while Fig. 7 shows the experiment pressure tap locations at the camera fairing and inlet bump regions. For reference, the nose of the aircraft is at axial station $x=2.419$ ", which is consistent with the aircraft model ru n in the experiment. In addition, the inlet mass flow rate ratio was defined as the ratio of the mass flow rate at the aerodynamic interface plane (AIP), $m_{2}$, to the theoretical capture mass flow rate, $m_{0}$. The capt u re mas s flow rate utilized the inlet throat area as the capture area, which does not accountfor the pre-compression surface of the in let bump diverter. Thus, some of the mass flow rate ratios reported in this paper exceed 1.00.

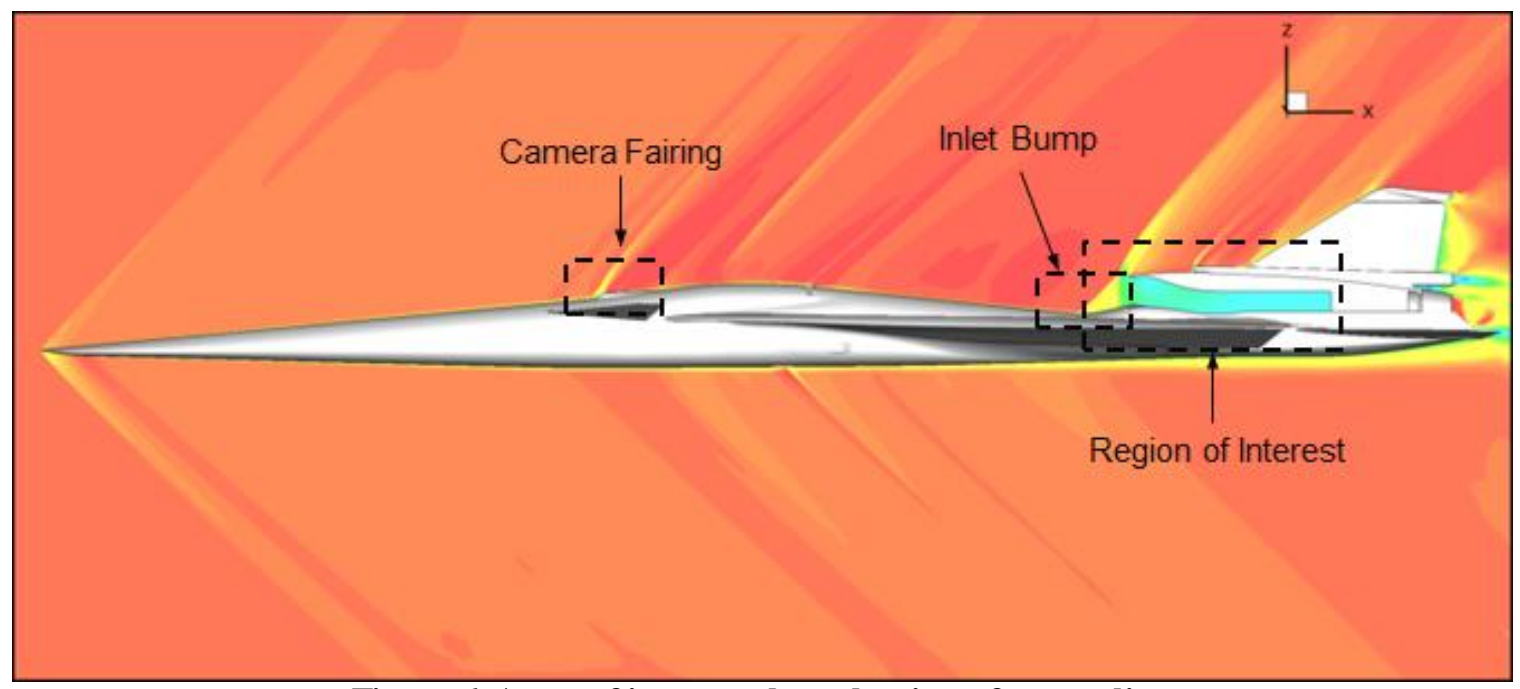

Figure 6. Areas of interest along the aircraft centerline.
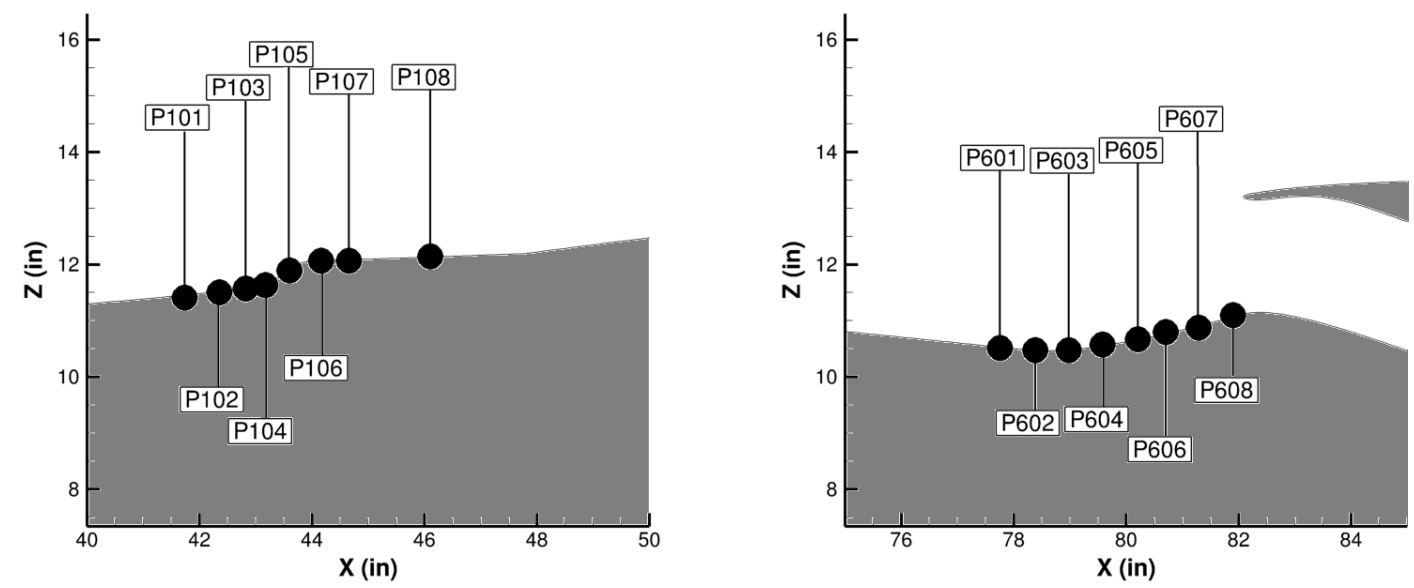

Figure 7. Experiment pressure tap locations along the aircraft centerline at the camera fairing region (left) and inlet bump region (right).

\section{A. Statistical Approach}

Paired t-tests were performed in order to help quantify the comparisons between the CFD solutions to thems elves and the experimental data. By definition, the pairedt-tests were performed on the differences between the data being compared and not the absolute values thems elves. For example, if a paired $t$-test were to be performed on data derived from CFD simulations and an experiment, shown in Table 3, the paired t-test would be performed on 
the differences between the two data sets, i.e. the third column of Table 3. Due to the limited number of CFD simulation data points available, thes tatistical comparisons presented in this paper utilized only 4-6 points per comparison. While this is notideal, it is the hope of the authors that the presented framework can be utilized for future CFD simulation comparisons.

Although the CFD simulations were run at the experimental conditions, the CFD simulations were not neces sarily run at the same inlet mass flow rate ratios as the experiment, therefore, curve fits were ap plied to the CFD data for a one-on-one comparis on with the experimental data. The curve fits were gen erated u sing a leas t squares error method to fit a 4th-order or lower polynomial to the CFD data as a function of the inlet mas s flow rate ratio, with an example curve fit shown in Fig. 8. CFD data with curve fits with an R-squared value less than 0.8 were omitted from the statistical comparis ons and the curve fits were used only for interpolation. Figures that utilize the curve fits have an asterisk at the end of each figure caption. The reader is cautioned that there is a difference between being statistically the same/different and being the same/different froman engineering pers pective. For example, two data sets might be close enough that from an engineering perspective they are the same, but statistically they are different due to the standard deviations being smaller than the average differencebe tween the two data sets. This will come into play in the following subsections.

Table 3: Example pressure data (as a function of the inlet mass flow rate ratio) at one of the inlet bump pressure taplocations.

\begin{tabular}{|c|c|c|}
\hline CFD & Experiment & CFD - Experiment \\
\hline 1645 & 1619 & 26 \\
\hline 1732 & 1700 & 32 \\
\hline 1870 & 1843 & 27 \\
\hline 2004 & 1962 & 42 \\
\hline
\end{tabular}

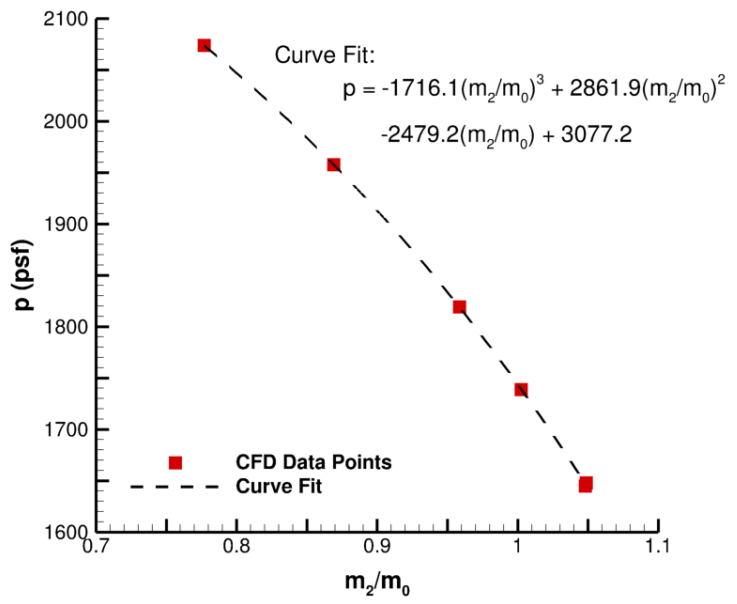

Figure 8. Example CFD curve fit at one of the inlet bump pressure taplocations.

\section{B. Cell Type and Grid Adaptation Metric}

Figure 9 shows the inlet Mach number contour on the unadapted tetrahedral boundary-layergrid at the Reading 1755 conditions $\left(M_{\infty}=1.46, \alpha=2.0^{\circ}\right)$ for an inlet mass flow ratio of 0.96 . It can be seen that FUN3D predicts a s mall separation region within the subsonic diffuser. This separation region is an artifact of the shockwave boundary-layer interaction occurring upstreamin the inlet bump region and therefore is sensitive tohow well the CFD code can predict the shockwave strength and location. Further, this separation region is shown to be greatly exaggerated after 8 adaptation cycles, shown in Fig. 10, when using the en gine axis-aligned linear pressure sensor. Note that during the adaptation process, the boundary-layer cells were "frozen" below a $y^{+}$of $\sim 300$ in order to permit a smooth transition from the viscous layers. Figure 11 shows the inlet Mach number contour on the unadapted pentah edral boundary-layer grid at the Reading 1755 conditions for an inlet mass flow ratio of 0.95 . Just like on the un a dapted tetrahedral grid, FUN3D predicts a small separation region within the subsonic diffuser. This too is greatly exaggerated after 8 adaptation cycles using the pressure boxobjective within the inlet subsonic diffu ser, s hown in 
Fig. 12. It should be noted that unlike the 8 adaptation cycle tetrahedral boundary-layer grid, the 8 adaptation cy cle pentahedral boundary-layer grid has an abrupt transition from the viscous regions. This is because FUN3D's adjoint-based grid adaptation refine/one library does not adapt pentahedral cells, and thus these cells were the only cells that were "frozen" during the adaptation process. In order to try to mitig ate this abrupt transition, the smoothed version of the unadapted pentahedral boundary-layer grid was run at the Reading 1755 conditions, with the Mach number contour for an inlet mass flow rate ratio of 0.95 shown in Fig. 13. In this case, FUN3D predicts a much larger separation compared to the previous unadapted grids, although it is still smaller than the ones predicted by the adapted grids. It was decided not to try adapting the pentahedral boundary-layer smooth grid as the previous results showed that adapting the grid would only increase the size of the separation region.

While comparing aircraft centerline Mach number contours is great for qualitative CFD solution comparis ons, it does not answer the question of how well each solution is correctly predicting the flow field, let alone pre dict ing inlet performance. To help answer these questions, the static pressure profiles at the camera fairing and inlet b u mp regions were plotted at various inlet mass flow rate ratios. Some of these are shown in Fig. 14 and Fig. 15 for the camera fairing region and Fig. 16 through Fig. 18 for the inlet bump region. The camera fairing region figures show that the pressure measurements at this location are insensitive to the inlet mass flow rate ratio. This is to be expected as the inlet is well downs tream of the camera fairing location. The inlet bump region figures show that the pres sure profiles are insensitive to the inlet mas s flow rate ratio only to about $x=80.2$ ", at which point, the pre ssure measurements tend to decrease as the mass flow rate ratio is increased. The exception to this is the CFD s olutions on the 8 adaptation cycle tetrahedral boundary-layer grid, which shows sensitivity to theinlet mas s flow rate ratio as far upstream as $x=79.6$ ". To further compare the CFD solutions and the experimental data, the static pres sure profiles at the camera fairing and inlet bump at an inlet mass flow rate ratio of 0.95 were plotted, as shown in Fig. 19. Note that the uncertainty in the experiment pressure data is $\pm 2.16 \mathrm{psf}$. It can be seen that the solutions from the pendahetral boundary-layer grids matched the experimental data better at the camera fairing compared to their tetrahedral boundary-layer grid counterparts. This trend shifts slightly downstreamat the in let bu mp as all CFD solutions tend to agree well with the experimental data, with the exception of the 8 ad aptation cy cle te trahedral boundary-layer grid. In terms of inlet performance, the 40-probe average total pressure recovery, shown in Fig. 20, was better predicted on the unadapted grids compared to their adapted counterparts. While qualitativ ely better predicted, could it be said that the average total pressure recoveries are statis tically the same? Based on the pairedttests, all of the CFD solutions with the exception of the solutions obtained on the 8 ad aptation cy cle tetrahedral boundary-layer grid statistically agree with the experimental data at the $95 \%$ confidence level.

To give a qualitative idea of inlet dis tortion, the computed 40-probe total pressure recovery contours at the A IP are shown in Fig. 21 through Fig. 23. These show that the size of the lower total pressure recovery region in the bottomportion of the inlet increased in the adapted cases compared to the unadapted cases. This trend is related to the increase in the separation region shown in the Mach number contour plots. Unlike the aircraft centerline Mach number contour plots, experimental data was available at the AIP. It is shown that the CFD first under predicts and then over predicts the size of the lower total pressure recovery region. To quantify the inlet distortion, ARP 1420 distortion parameters ${ }^{19}$ were computed and shown in Fig. 24. Unlike the total pressure recovery, qualitatively the inlet distortion was not well predicted by any of the CFD solutions. However, only the circumferential inlet distortion computed on the unadapted grids were statistically the same as the experimental data at the $95 \%$ confidence level.

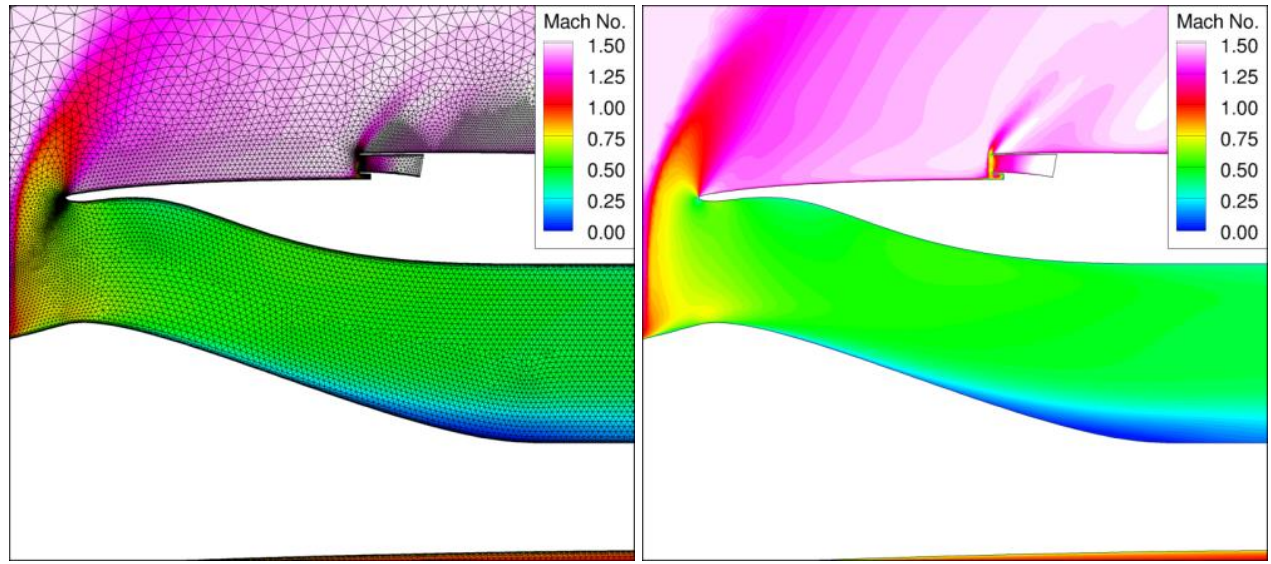

Figure 9. Mach number contours for the unadapted tetrahedral boundary-layer grid, $m_{2} / m_{0}=0.96$. 

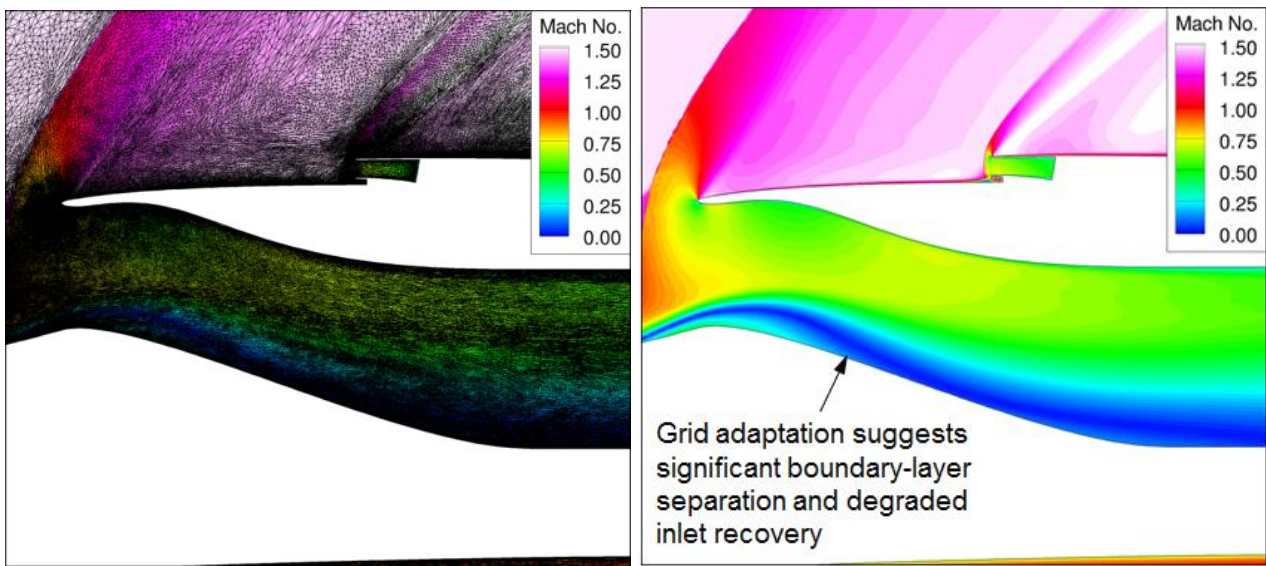

Figure 10. Mach number contours for the 8 adaptation cycle tetrahedral boundary-layer $\mathrm{grid}, \mathrm{m}_{2} / \mathrm{m}_{0}=0.93$.

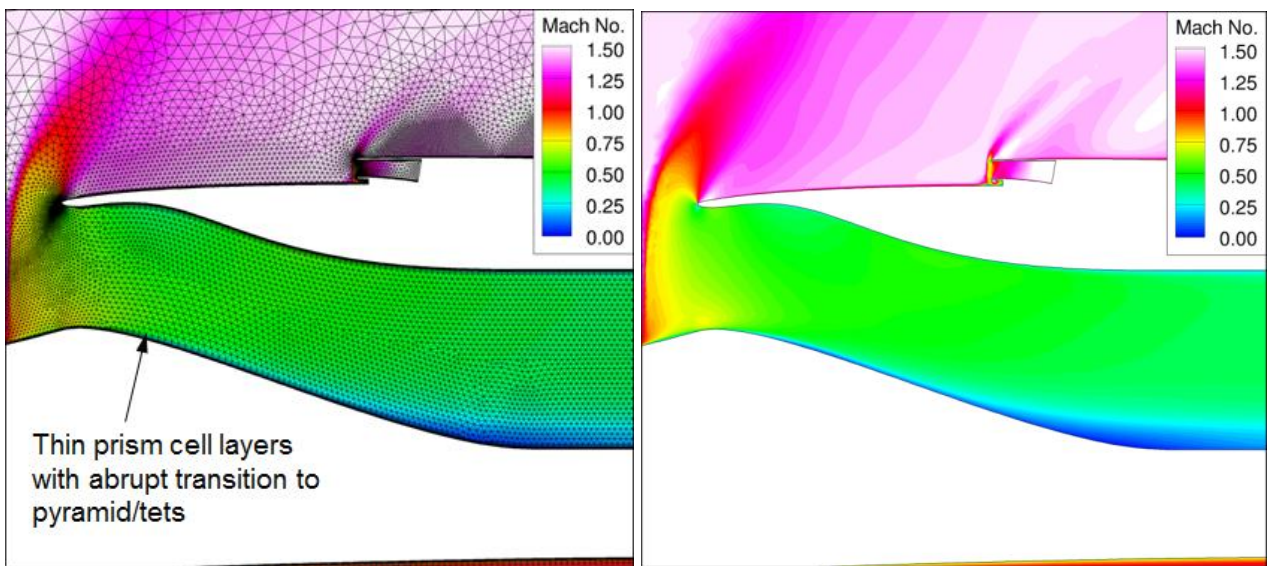

Figure 11. Mach number contours for the unadapted pentahedral boundary-layer grid, $m_{2} / m_{0}=0.95$.
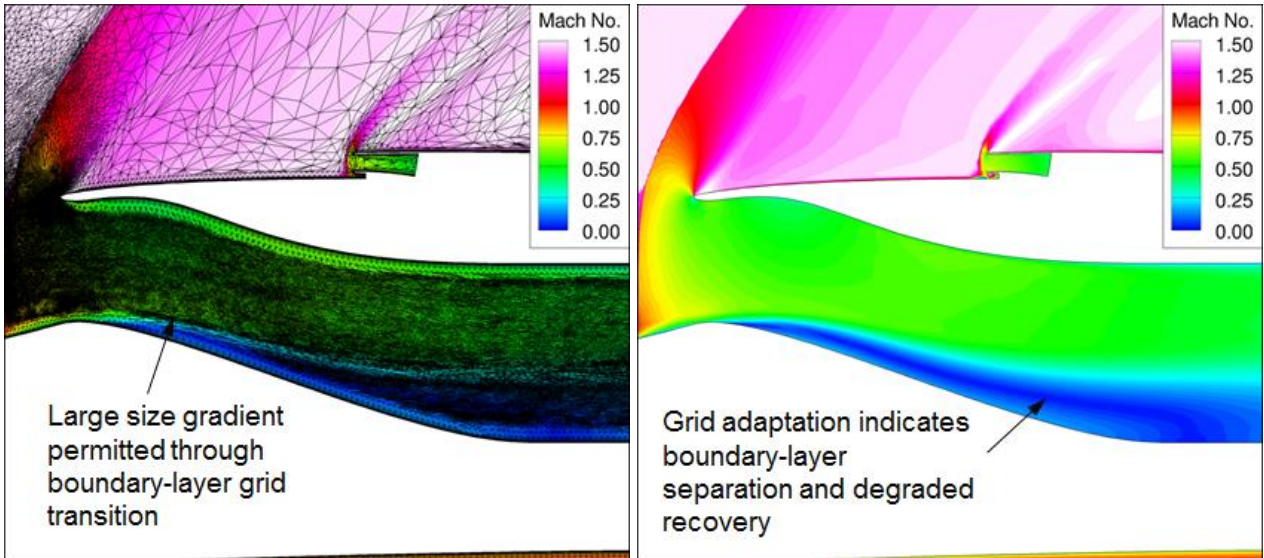

Figure 12. Mach number contours for the 8 adaptation cycle pentahedral boundary-layer grid, $m_{2} / m_{0}=0.94$ 


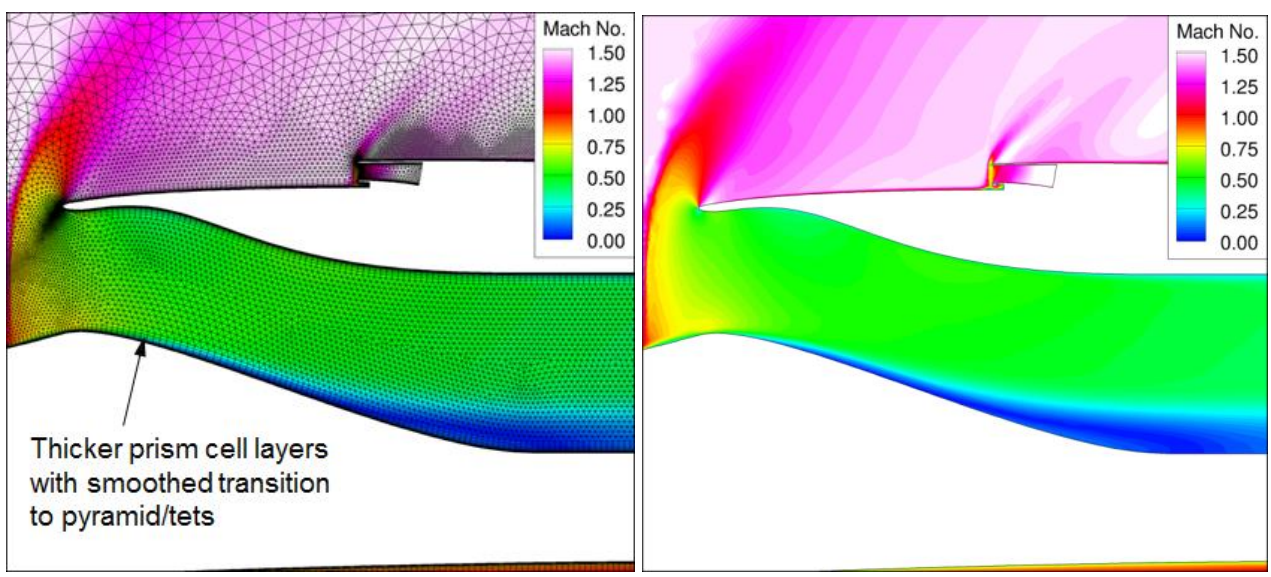

Figure 13. Mach number contours for the unadapted pentahedral boundary-layer smooth grid, $m_{2} / m_{0}=0.95$.
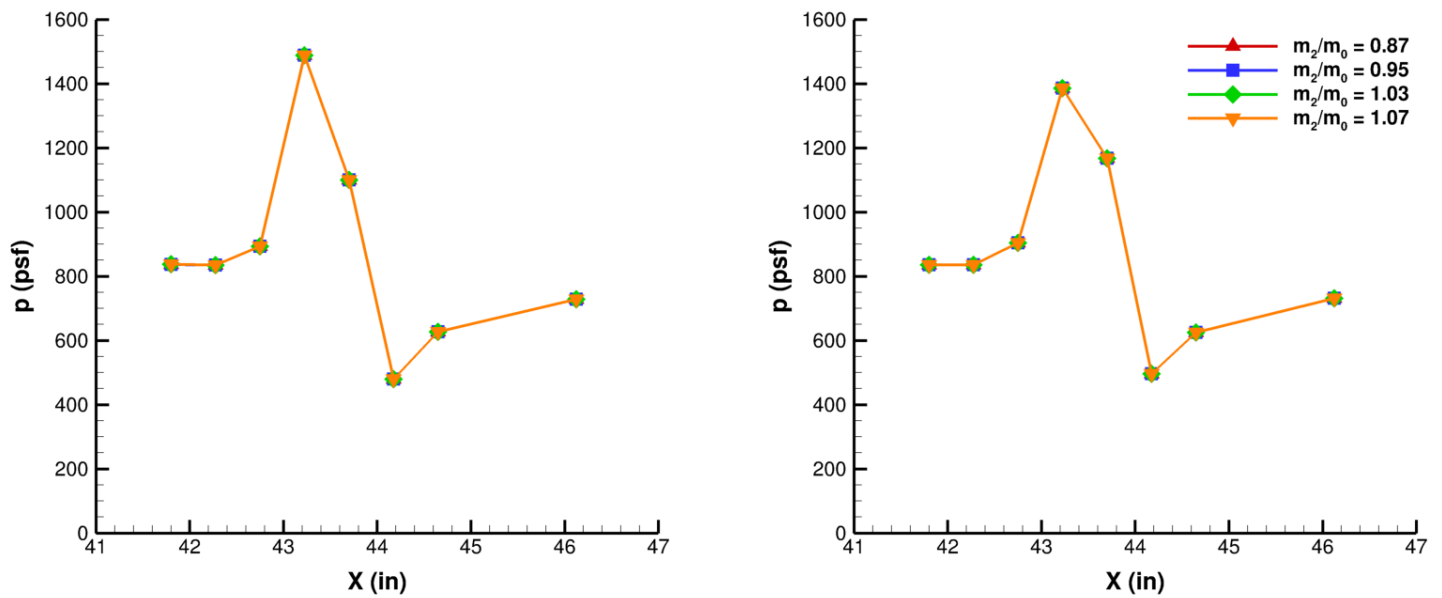

Figure 14. Static pressure profiles along the camera fairing for the unadapted (left) and 8 adaptation cycle (right) tetrahedral boundary-layer grids for $M_{\infty}=1.46, \alpha=2.0^{\circ} .^{*}$

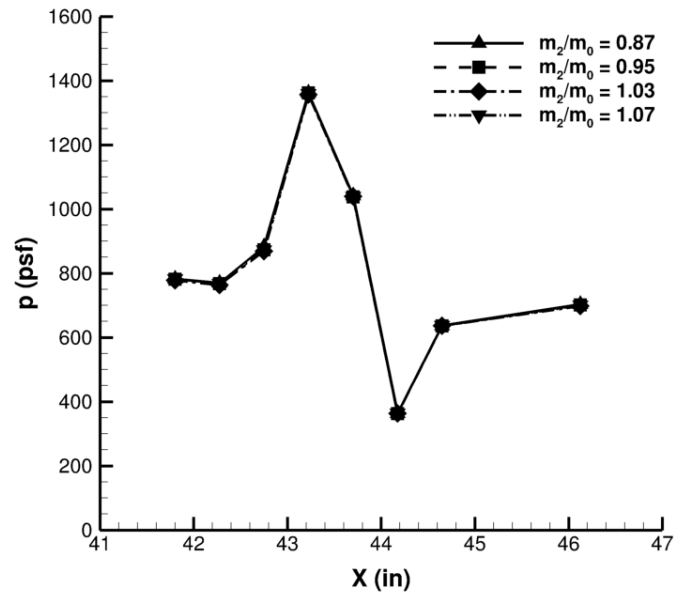

Figure 15. Static pressure profiles along the camera fairing for the experiment at $M_{\infty}=1.46, \alpha=2.0^{\circ}$. 

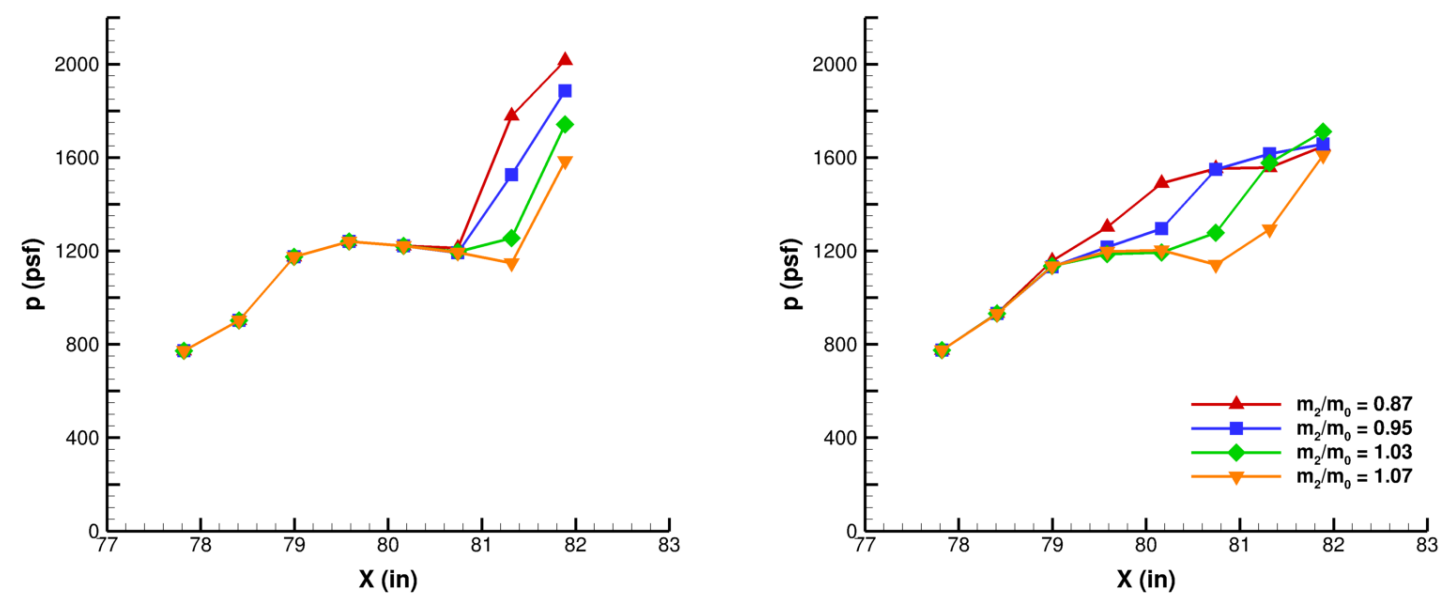

Figure 16. Static press ure profiles along the inlet bump for the unadapted (left) and 8 adaptation cycle (right) tetrahedral boundary-layer grids for $M_{\infty}=1.46, \alpha=2.0^{\circ} .^{*}$
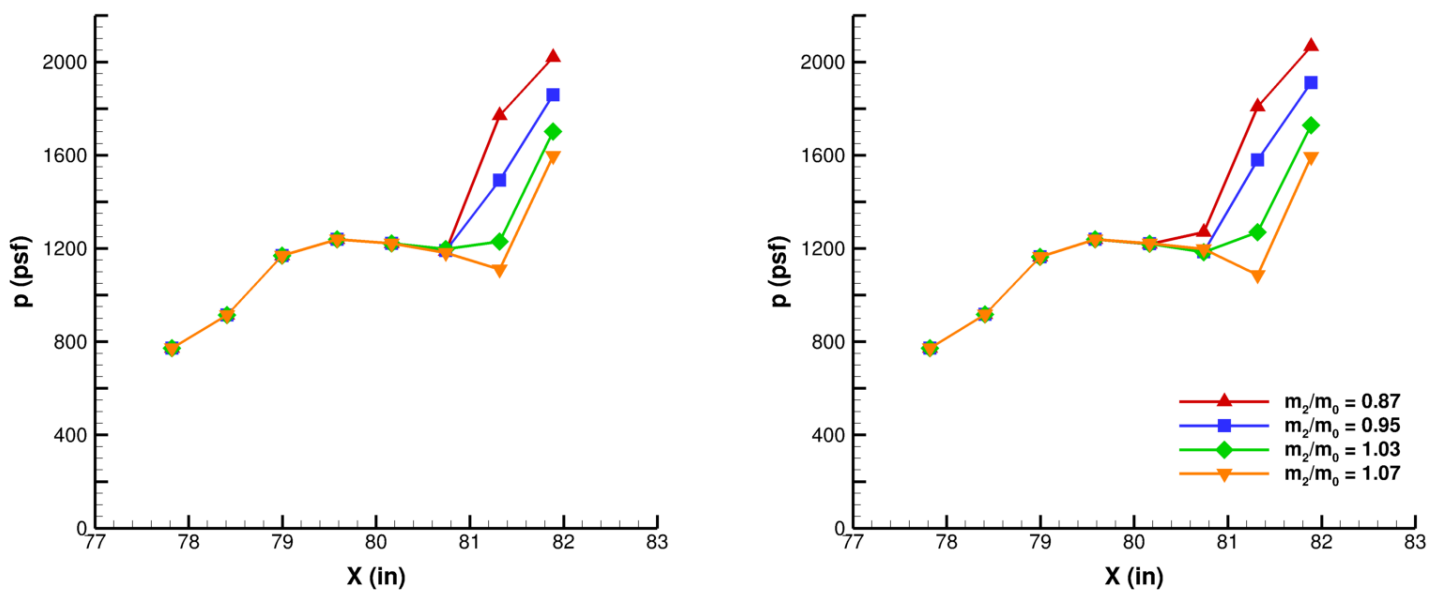

Figure 17. Static press ure profiles along the inlet bump for the unadapted (left) and 8 adaptation cycle (right) pentahedral boundary-layer grids for $M_{\infty}=1.46, \alpha=2.0^{\circ} . *$

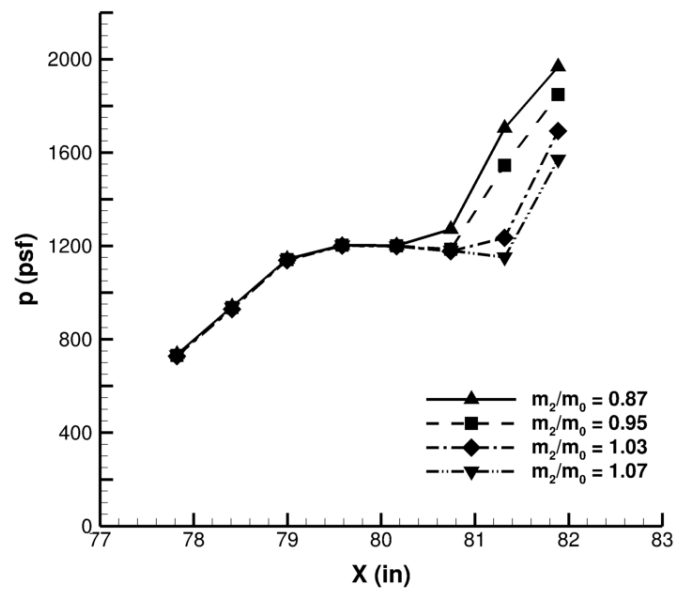

Figure 18. Static press ure profiles along the inlet bump for the experiment at $M_{\infty}=1.46, \alpha=2.0^{\circ}$. 

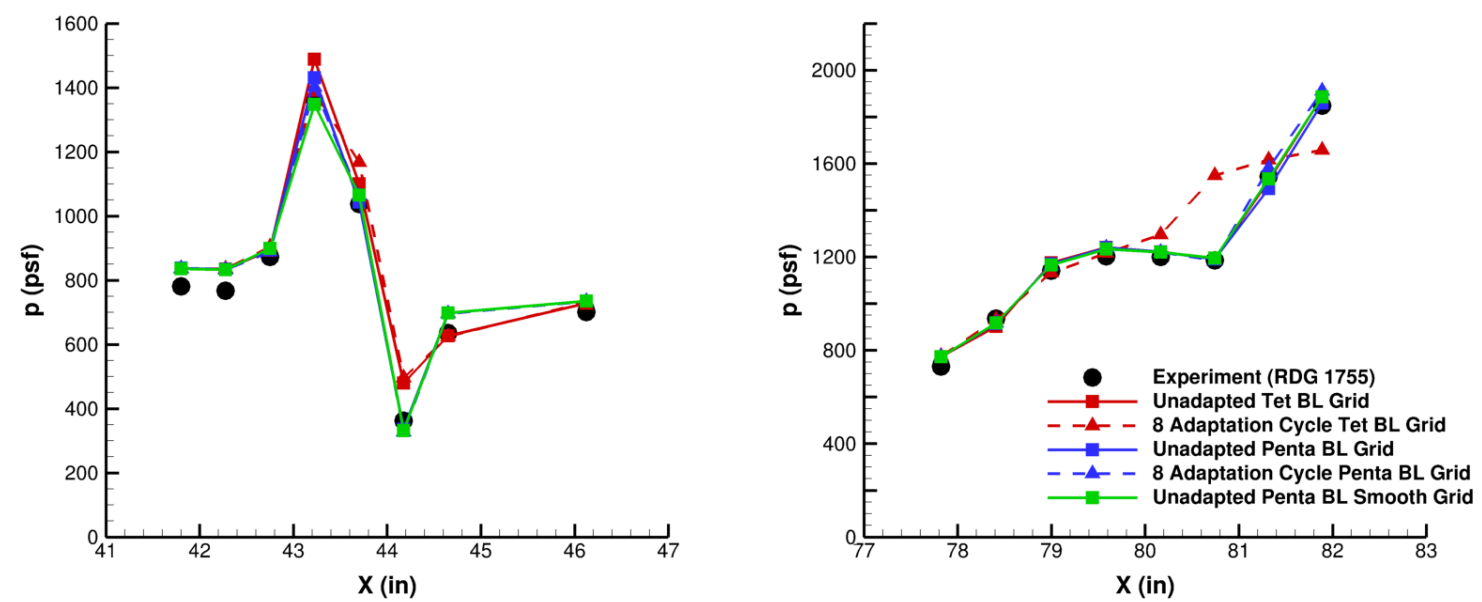

Figure 19. Static press ure profiles along the camera fairing (left) and inlet bump (right) for $M_{\propto}=1.46$, $\alpha=2.0^{\circ}, m_{2} / m_{0}=0.95 * *$

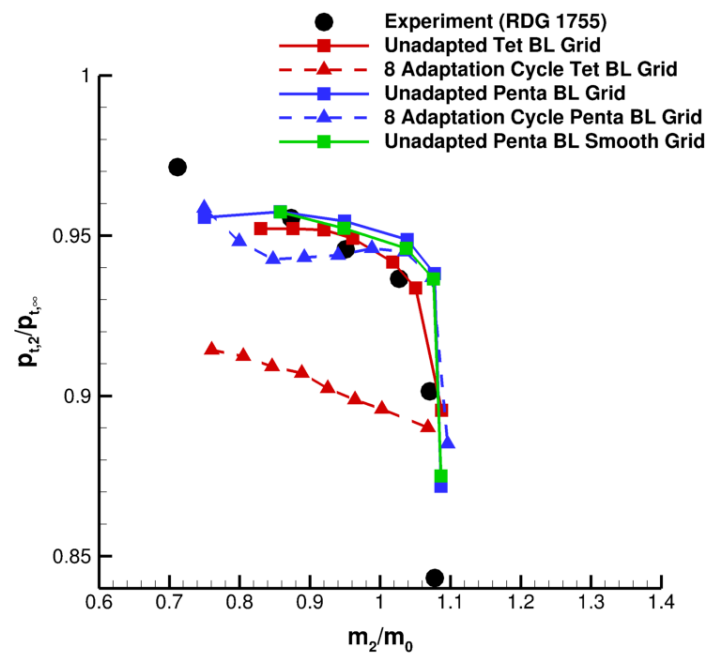

Figure 20.40-probe total pressure recovery plot for $M_{\propto}=1.46, \alpha=2.0^{\circ}$.
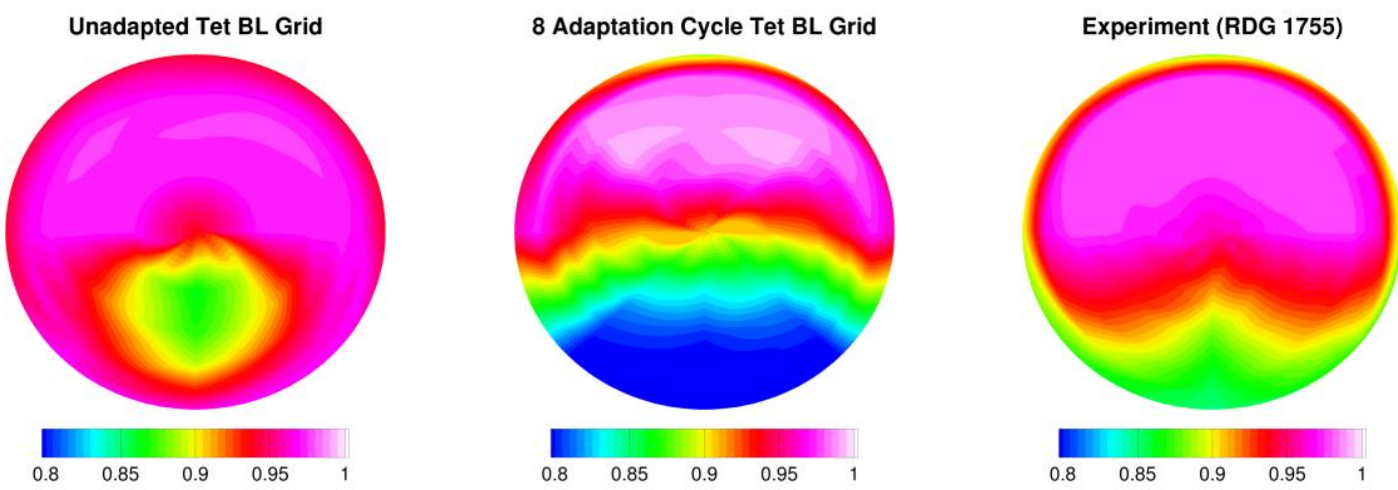

Figure 21.40-probe total pressure recovery contours for the unadapted (left) and 8 adaptation cycle (middle) tetrahedral boundary-layer grid compared to the experiment (right) at $M_{\infty}=1.46, \alpha=2.0^{\circ}$. 

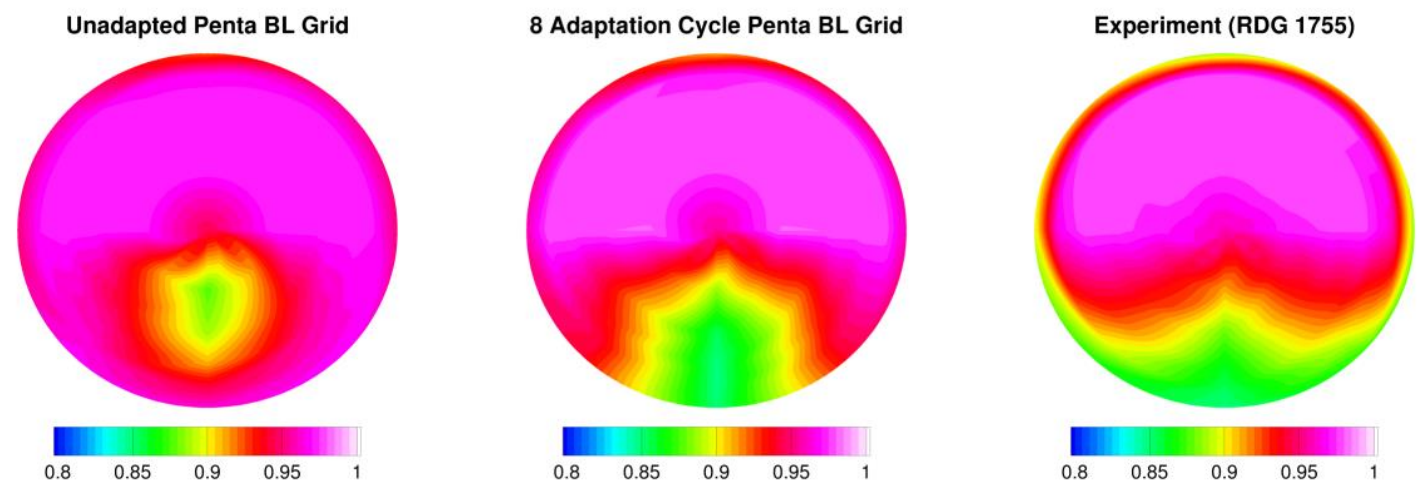

Figure 22.40-probe total pressure recovery contours for the unadapted (left) and 8 adaptation cycle (middle) pentahedral boundary-layer grid compared to the experiment (right) at $M_{\propto}=1.46, \alpha=2.0^{\circ}$.
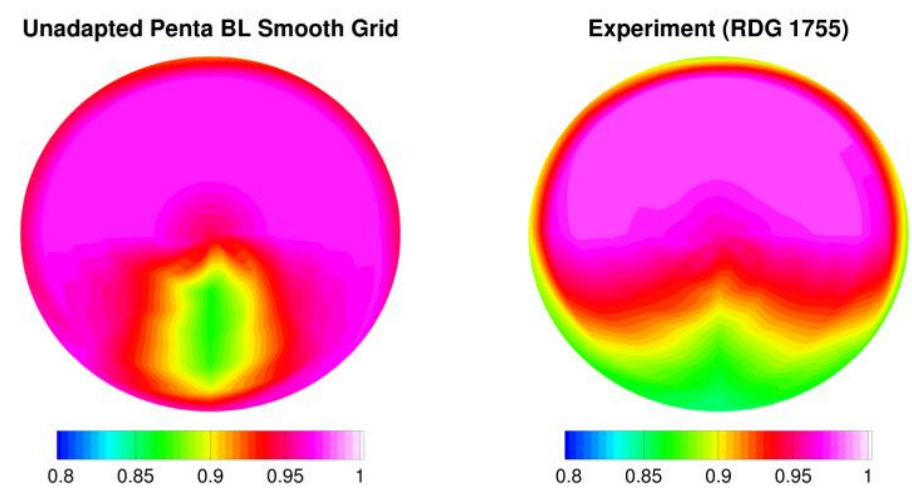

Figure 23.40-probe total pressure recovery contours for the unadapted pentahedral boundary-layer smooth grid(left) and the experiment (right) at $M_{\alpha}=1.46, \alpha=2.0^{\circ}$.

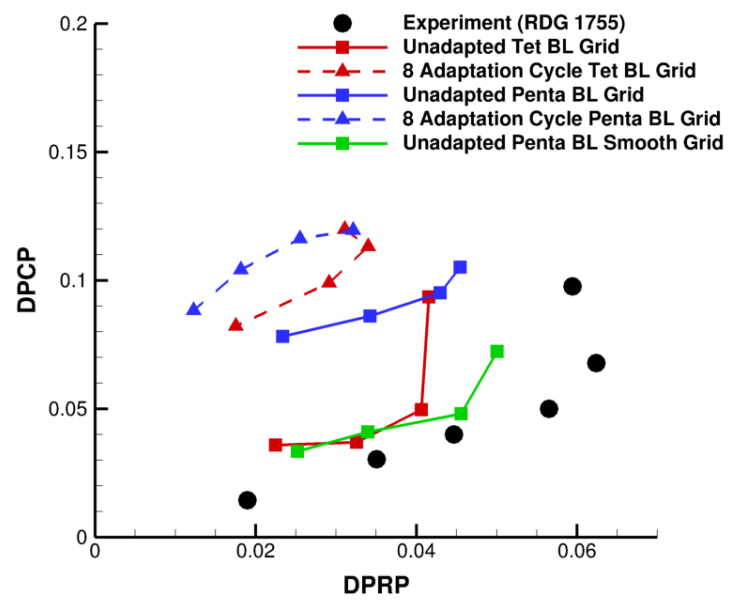

Figure 24. Inlet distortion plot for $M_{\propto}=1.46, \alpha=2.0^{\circ} .^{*}$

\section{Number of Adaptation Cycles}

While the number of adaptation cycles was increased from 8 to 16 for this sub-study, it should be noted that the target number of nodes added per adaptation cycle was decreased from $1 \times 10^{6}$ to $2 \times 10^{5}$ in order to reduce the grid size of the 16 adaptation cycle grids. Thus the 8 adaptation cycle grids presented in this subsection had approximately $25-54 \%$ fewer nodes compared to theircounterparts in the previous subsection. Figure 25 and Fig. 
26 show the aircraft centerline Mach number contours for the 8 and 16 adaptation cycle tetrahedral boundary-layer grids at the Reading 1755 conditions $\left(M_{\infty}=1.46, \alpha=2.0^{\circ}\right)$ while Fig. 27 and Fig. 28 show the aircraft centerline Mach number contours for the 8 and 16 adaptation cycle pentahedral boundary-layer grids at the Reading 1755 conditions. These figures show that there is very little difference in the flow field between 8 and 16 adaptation cy cles. Also, they predict a large separation region in the subsonic diffuser, which is consistent with the separation regions predicted on the adapted grids shown in Fig. 10 and Fig. 12.

Figure 29 shows static pressure profiles at the camera fairing and the inlet bump at the Reading 1755 condition s for the unadapted, 8 adaptation cycle, and 16 adaptation cy cle tetrahedral and pentahedral boundary-layerg rid s. It can be shown that the pressure profiles at the camera fairing generally agree with each other. Figure 29 also s hows that the pressure profiles at the in let bump generally agree with each other with the exception of the second to las $t$ pressure station $(x=81.3$ ").

In terms of the 40-probe average total pres sure recovery, shown in Fig. 30, both the pentahedral boundary-lay er and tetrahedral boundary-layer grids showed asymptotically decreasing total pressure reco very as the number of adaptation cycles was increased. This was more pronounced on the tetrahe dral boundary-layer grids than the pentahedral boundary-layer grids. The trend that the 40-probe average total pre ssure recovery a smp totically decreased as the number of adaptation cycles increased suggests that while adjoint-based grid ad aptation will converge to a value for the 40-probe average total pressure recovery, that value will most likely not be the same value as that provided by experimental data. Further insightcan be gained by looking at the computed AIP 40-probe total pres sure recovery contour plots, shown in Fig. 31 for the tetrahedral boundary-layergrids andFig. 32 for the pentahedral boundary-layergrids. It can be seen that the total pressure recovery does not change signific antly between 8 and 16 adaptation cycles while also over predicting the region of lower total pressure recovery in the bottomportion of the inlet. This is consistent with what was seen in the adapted grid solutions in Fig. 21 and Fig. 22.

Although using the adjoint-based solver in FUN3D resulted in poor agreement with the experimental data for these cases, the adjoint solver itself might not be the is sue. In FUN3D, error estimate s are computed after each adaptation cycle using the Vendittierror estimate ${ }^{20}$. The computation of this error estimate, called the re maining adaptation error, is summarized in Eq. (1).

$$
\begin{aligned}
\text { Remaining AdaptationError }= & ([\text { Flow Residual on Embedded Mesh }] \times[\text { Adjoint Interpolation Error }])+ \\
& ([\text { Adjoint Residual on Embedded Mesh }] \times[\text { Flow Interpolation Error }])
\end{aligned}
$$

Examining the remaining adaptation error for the pentahedral boundary-layer grid in Fig. 33 shows that the remaining adaptation error is actually increasing over the course of the first 5 adaptation cycles and then steadies out to a relatively high value (on the order of $10^{3}$ ). This is des pite the fact that the flow residuals over the course of each adaptation cycle are shown to level out. Figure 33 also shows an example of the flow residuals during the $3^{\text {rd }}$ adaptation cycle. Similar trends in the flow residuals were seen during the other adaptation cycles and the remaining adaptation error followed a similar trend for the tetrahedral boundary-layer grid. A possible reas on for the lack of convergence of the remaining adaptation error is that the error estimation is using noisy data. The noisy data is most likely due to poor convergence of the flow equations, which in turn is due to numerical instability or physical unsteadiness of the flow field. 

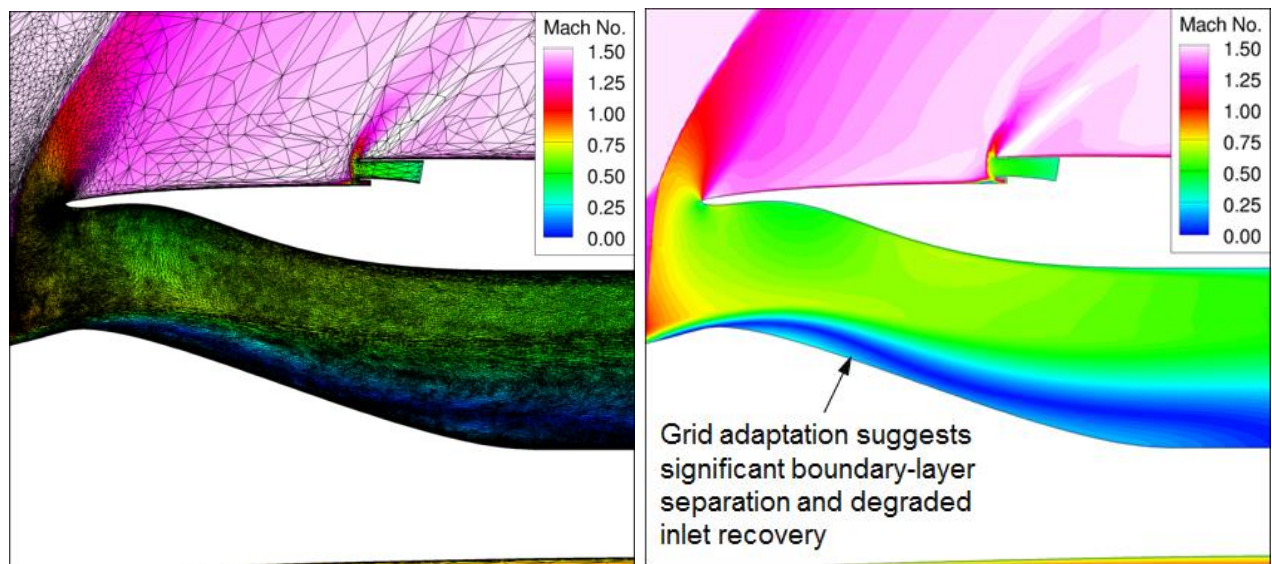

Figure 25. Mach number contours for the 8 adaptation cycle tetrahedral boundary-layer $\mathrm{grid}, \mathrm{m}_{2} / \mathrm{m}_{0}=0.97$.
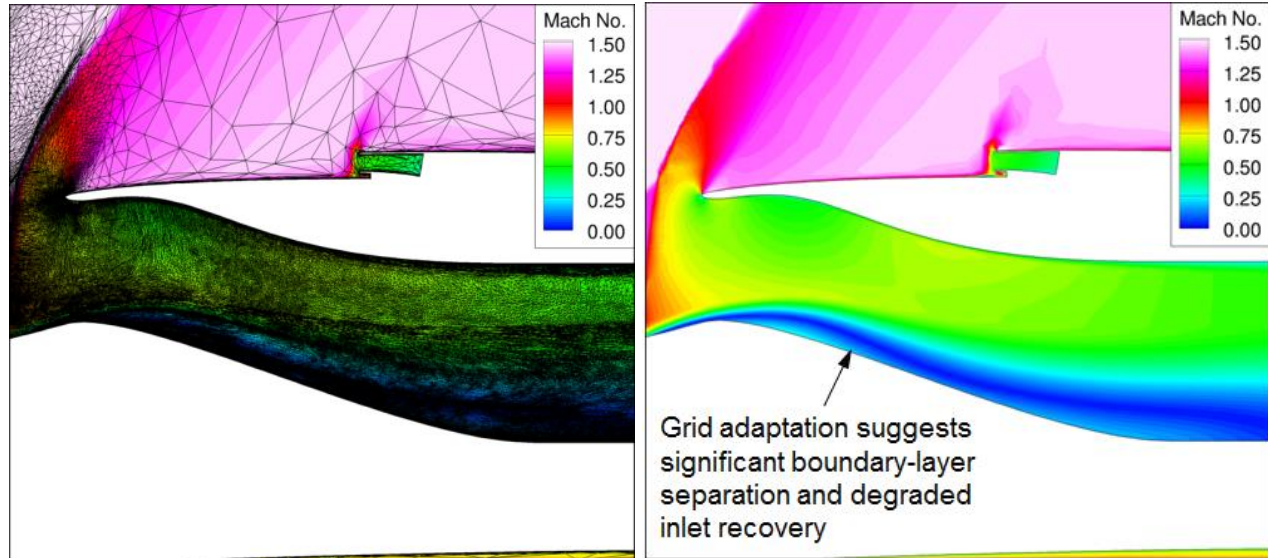

Figure 26. Mach number contours for the 16 adaptation cycle tetrahedral boundary-layer grid, $m_{2} / m_{0}=0.97$.
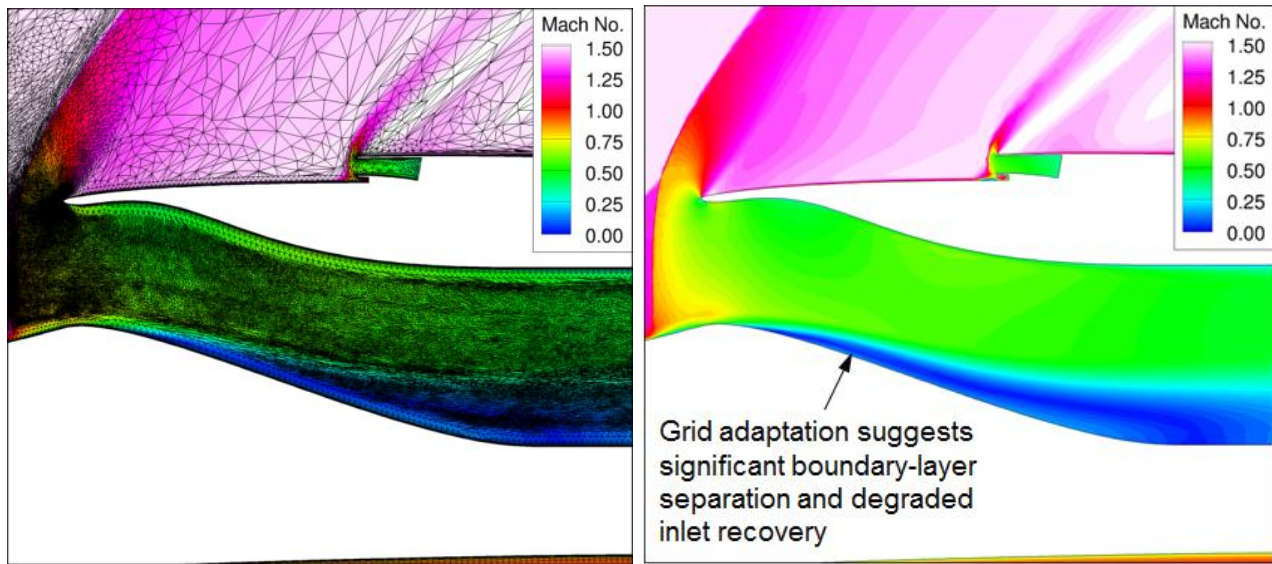

Figure 27. Mach number contours for the 8 adaptation cycle pentahedral boundary-layer grid, $m_{2} / m_{0}=0.99$. 


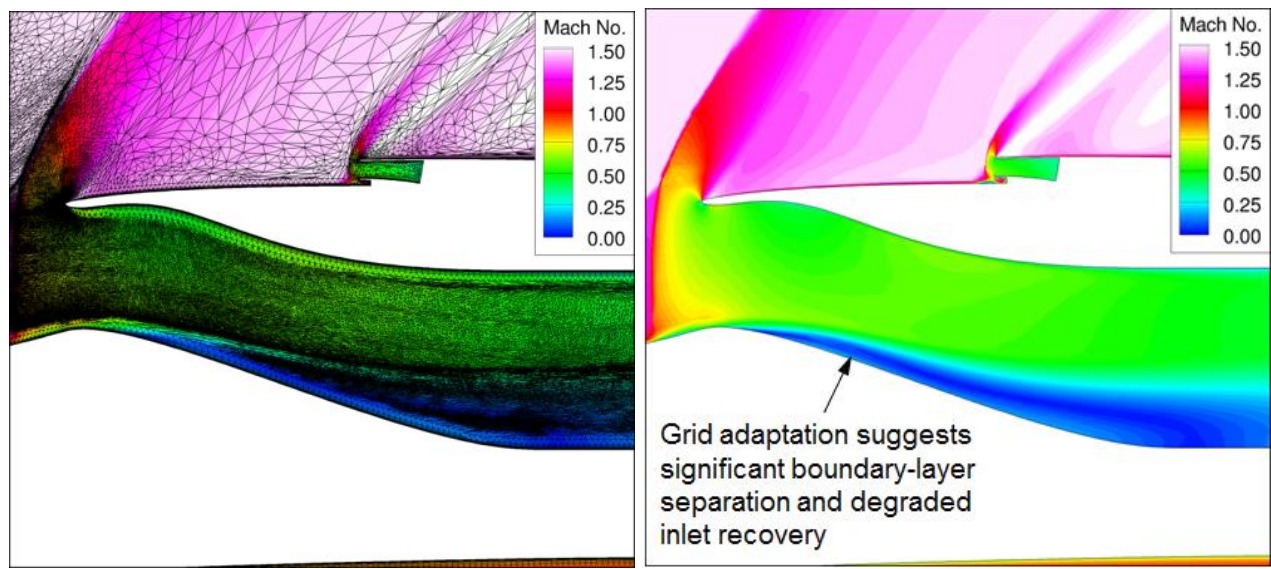

Figure 28. Mach number contours for the 16 adaptation cycle pentahedral boundary-layer grid, $m_{2} / m_{0}=0.99$.
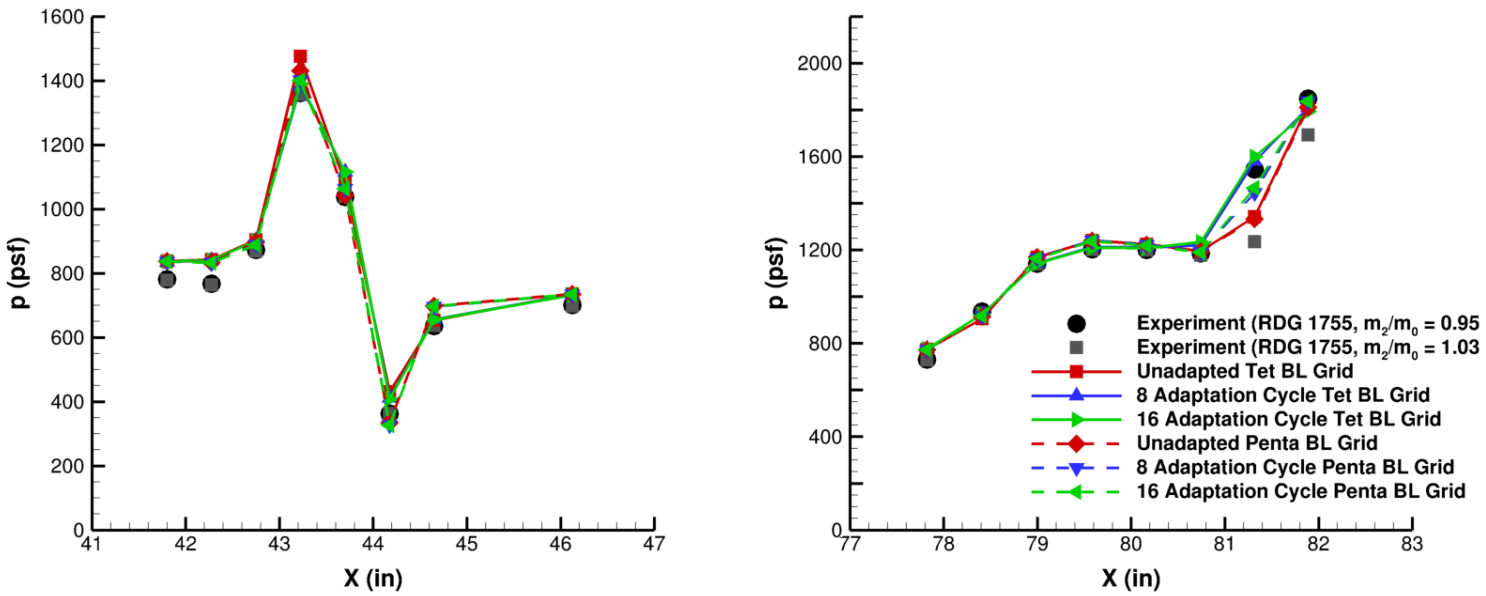

Figure 29. Static press ure profiles along the camera fairing (left) and inlet bump (right) for $M_{\infty}=1.46$, $\boldsymbol{\alpha}=\mathbf{2 . 0}$.
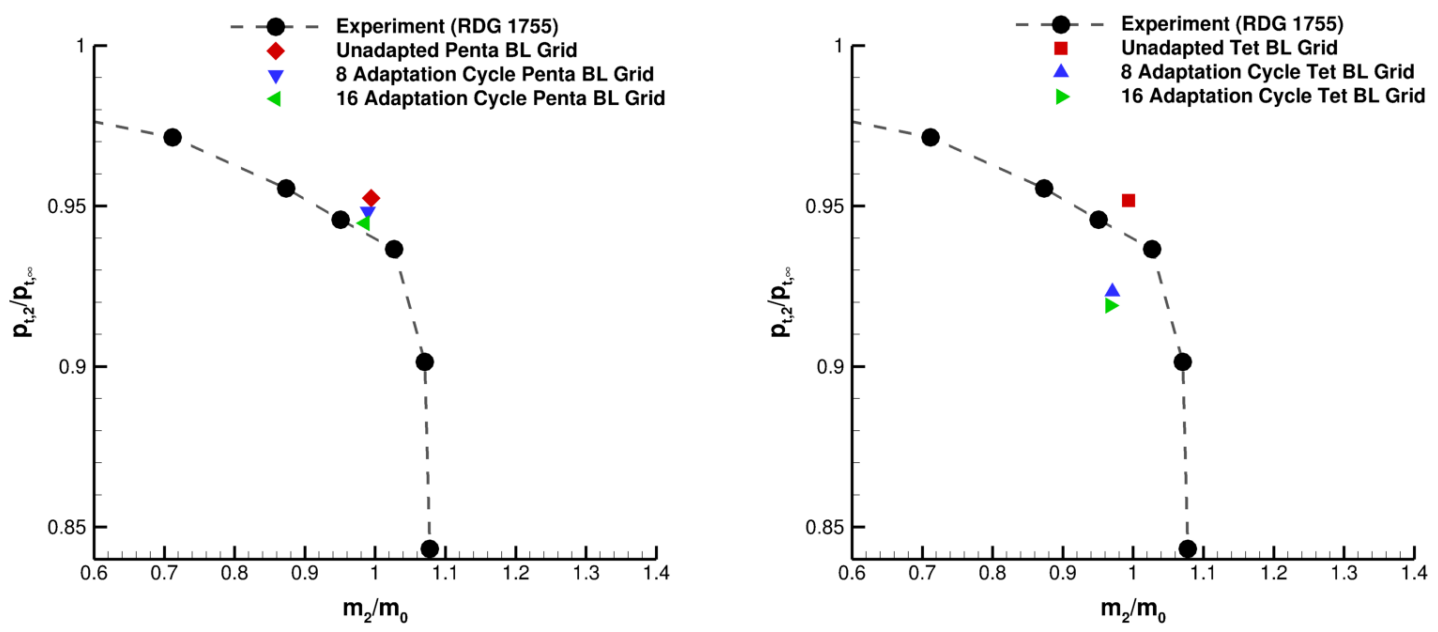

Figure 30.40-probe total pressure recovery plots on the pentahedral boundary-layer grids (left) and on the tetrahedral boundary-layer grids (right) for $M_{\infty}=1.46, \alpha=2.0^{\circ}$. 

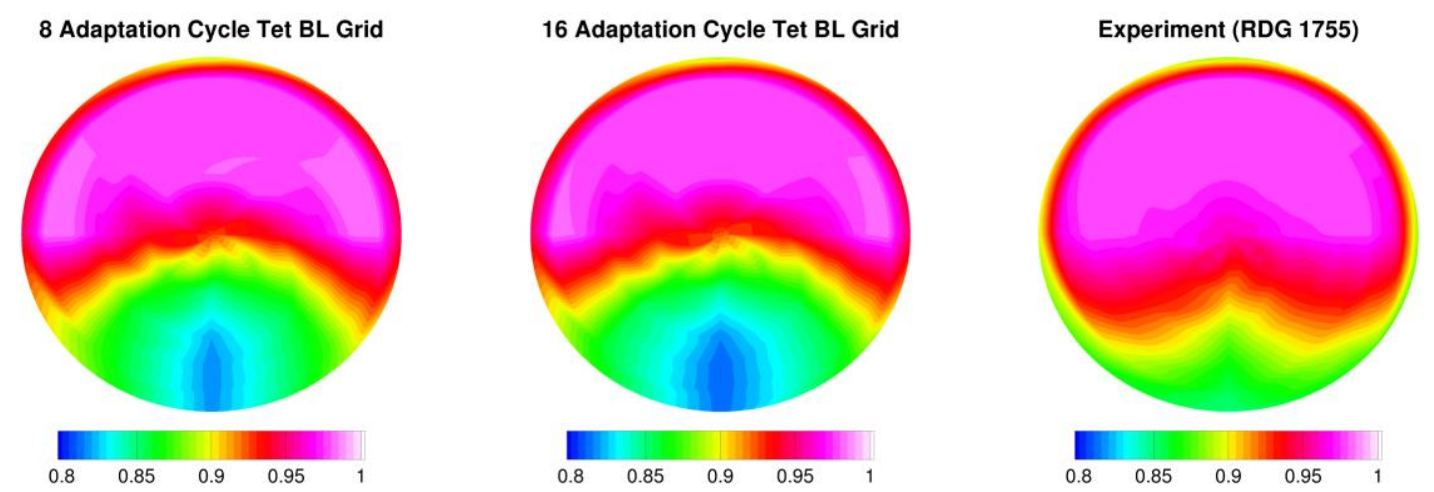

Figure 31.40-probe total pressure recovery contours for the 8 adaptation cycle (left) and 16 adaptation cycle (middle) tetrahedral boundary-layer grids compared to the experiment (right) at $M_{\infty}=1.46, \alpha=2.0^{\circ}$.
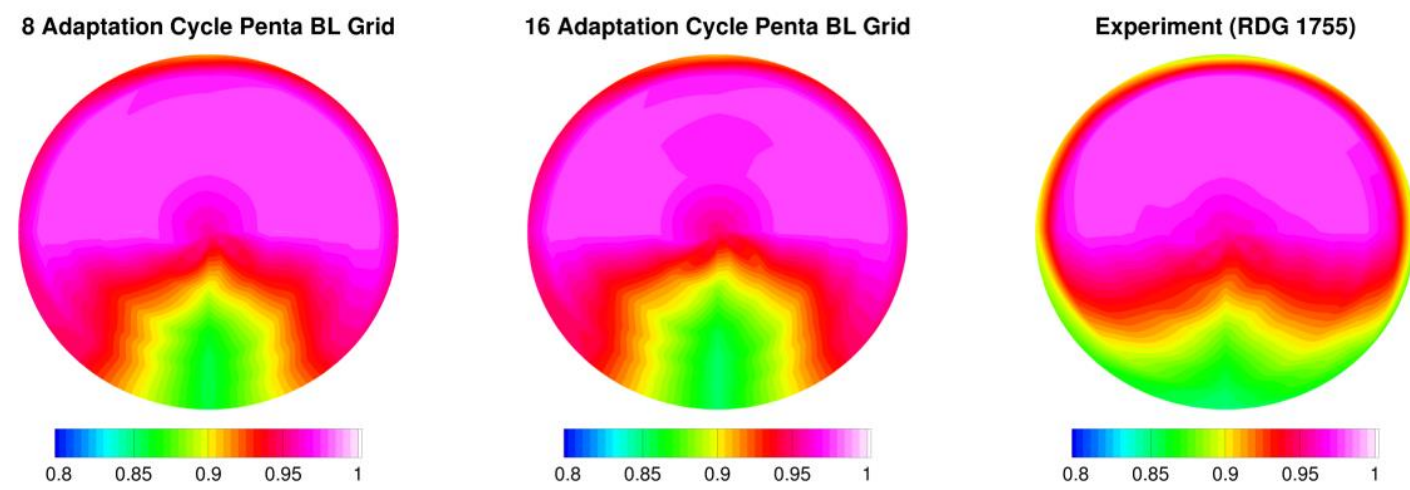

Figure 32.40-probe total pressure recovery contours for the 8 adaptation cycle (left) and 16 adaptation cycle (middle) pentahedral boundary-layer grids compared to the experiment (right) at $M_{\alpha}=1.46, \alpha=2.0^{\circ}$.
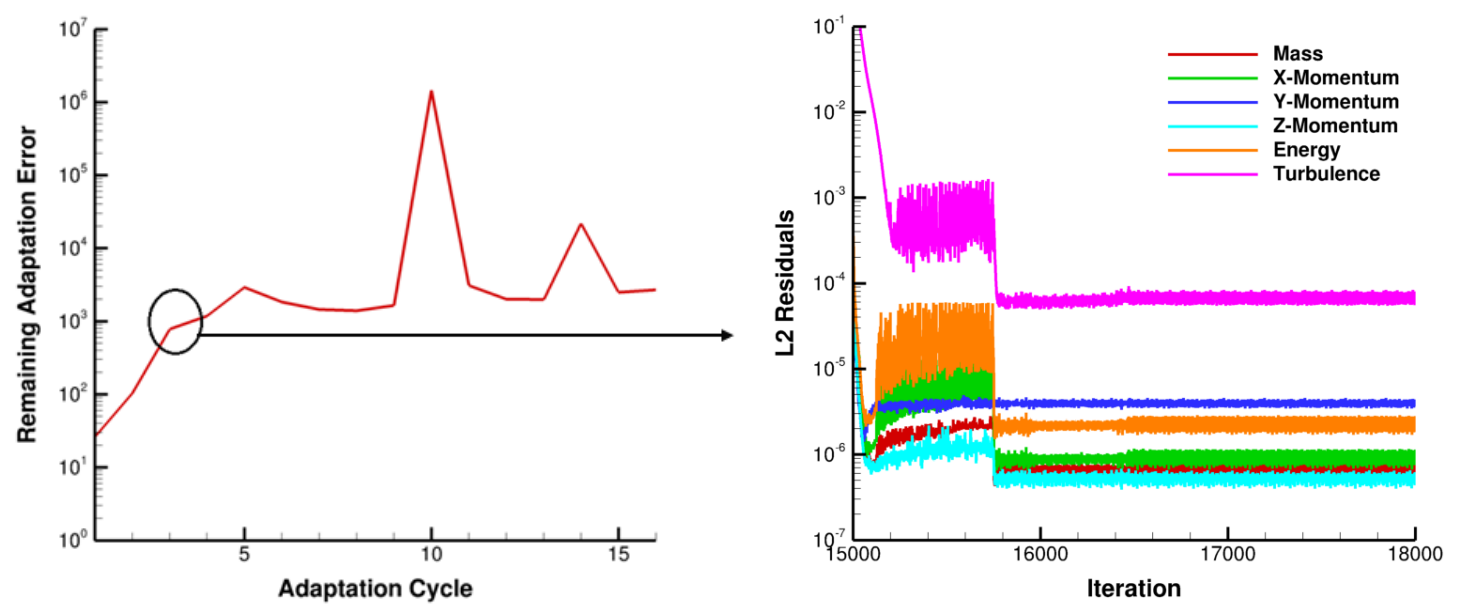

Figure 33. Remaining adaptation error (left) and $3^{\text {rd }}$ adaptation cycle flow residuals (right) for the pentahedral boundary-layer grid.

\section{Manually RefinedGrids}

Figure 34 and Fig. 35 show the Mach number contour plots along the aircraft centerline for the Reading 1755 conditions $\left(M_{\infty}=1.46, \alpha=2.0^{\circ}\right)$ on the manually refined grids. Just like on the adapted grids, the CFD solutions predict a large separation region within the subsonic diffuser, regardless of the cell type within/around the boundary- 
layer regions. Figure 36 shows the static pressure profiles at the camera fairing and inlet bump for the reading 1755 conditions on the manually refined grids. For comparis on, the solutions on the unadapted and 16 adapt at ion cy cle grids were included in the plots. The figure shows that the manually refined grids tend to agree with the unadapted and 16 adaptation cycle grids at the camera fairing with the exce ption of the fourth upstrea m location ( $x=43.2$ "), where the manually refined grids predict a lower pres sure than the 16 adaptation cycle grid. The figure als o s hows that the CFD solutions on the manually refined grids tend to agree with the other CFD solutions at the in let bu mp with the exception of the second to last downstream location $(x=81.3$ "). At this location, the pre dicted pressure measurements on the manually refined grids matches closely with thepressure me as urements predicted on the unadapted grids.

Figure 37 shows the 40-probe average total pressure recovery for the manually refined grids along with the 40probe average total pres sure recoveries for the unadapted and 16 adaptation cycle grids. Interestin gly, the fig u re shows that the average total pressure recovery on the manually refined pentahedral boundary-layer grid agrees with the unadapted pentahedral boundary-layer grid while the average total pressure recovery on the man u ally refined tetrahedral boundary-layer grid falls in between the unadapted and 16 adaptation cycle tetrahedral bo und ary -layer grids. One reas on for this dis crepancy between the two different cell-type grids could be that the phenomena that the total pressure recovery decreases as ymptotically as the adaption cycle (and thus the grid size) is increas ed was more pronounced on the tetrahedralboundary-layergrids than the pentahedralboundary-layerg rids. This would imply that one would expect a greater disagreement between the 40-probe average total pressure recoveries computed from the tetrahedral boundary-layer grids compared to those computed from the pentahed ral bou ndarylayer grids. Figure 38 and Fig. 39 show the 40-probe total pres sure recovery contour plots for the manually refined grids compared to the unadapted and 16 adaptation cycle grids. It can be seen that the manually refine d grid s are predicting a region of lower total pres sure recovery that is sized in between the equivalent regions predicted on the unadapted and 16 adaptation cycle grids. This makes sense as the number of nodes at the AIP on the man ually refined grids were greater than the number of nodes on the unadapted grids but less than the number of nodes on the 16 adaptation cycle grids.

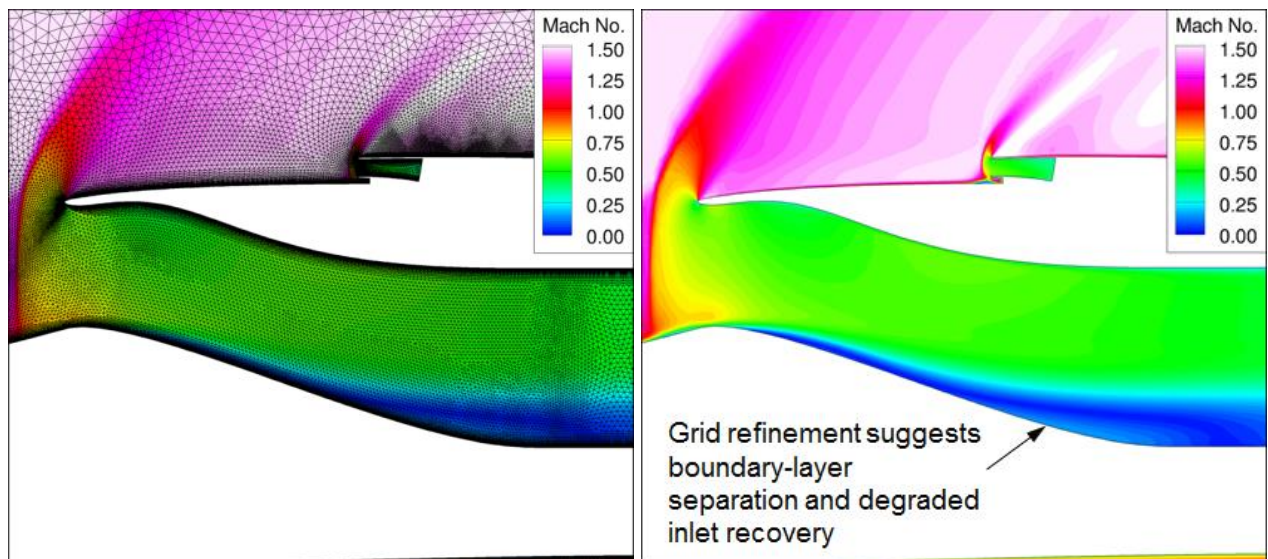

Figure 34. Mach number contours for the manually refined tetrahedral boundary-layer grid, $m_{2} / m_{0}=0.99$. 


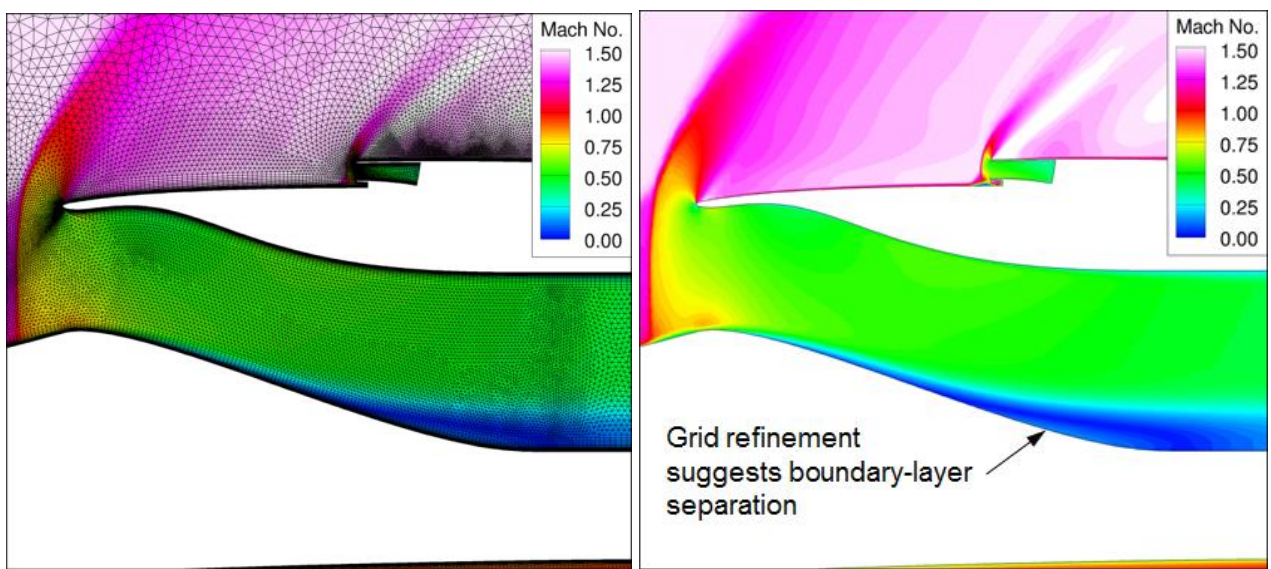

Figure 35. Mach number contours for the manually refinedpentahedral boundary-layer grid, $m_{2} / m_{0}=1.00$.
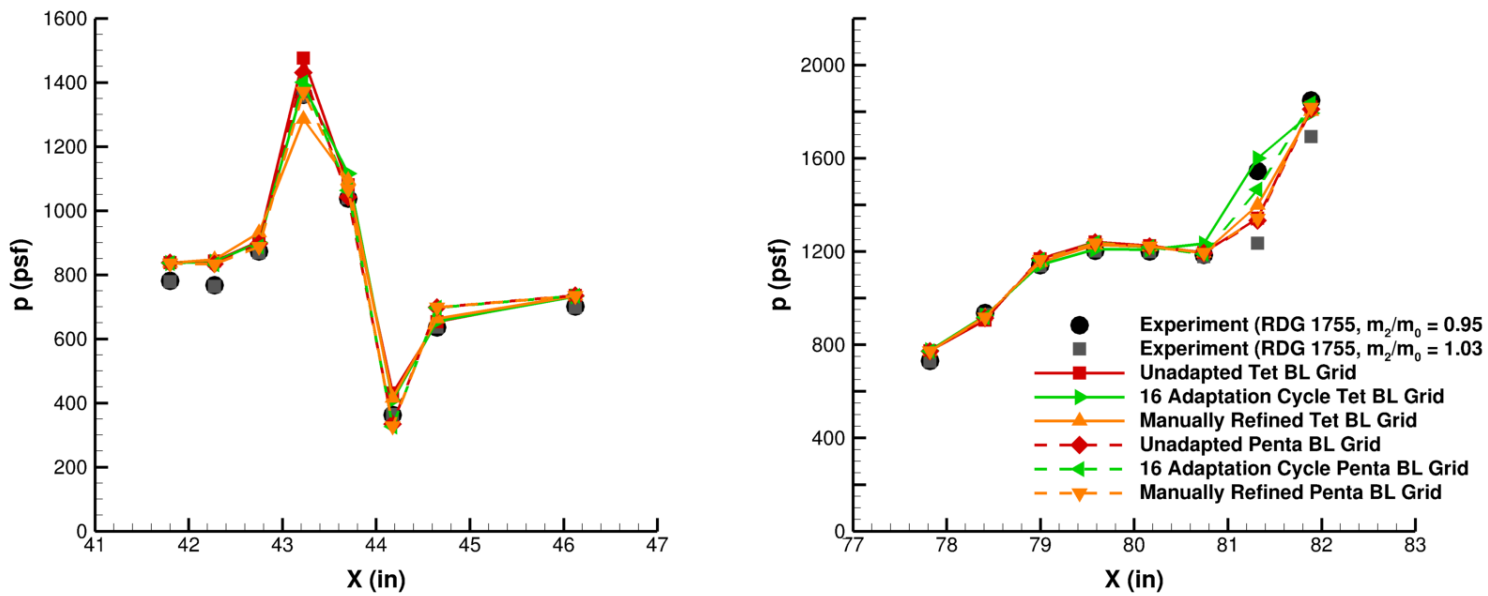

Figure 36. Static press ure profiles al ong the camera fairing (left) and inlet bump (right) for $M_{\propto}=1.46$, $\alpha=2.0^{\circ}$.
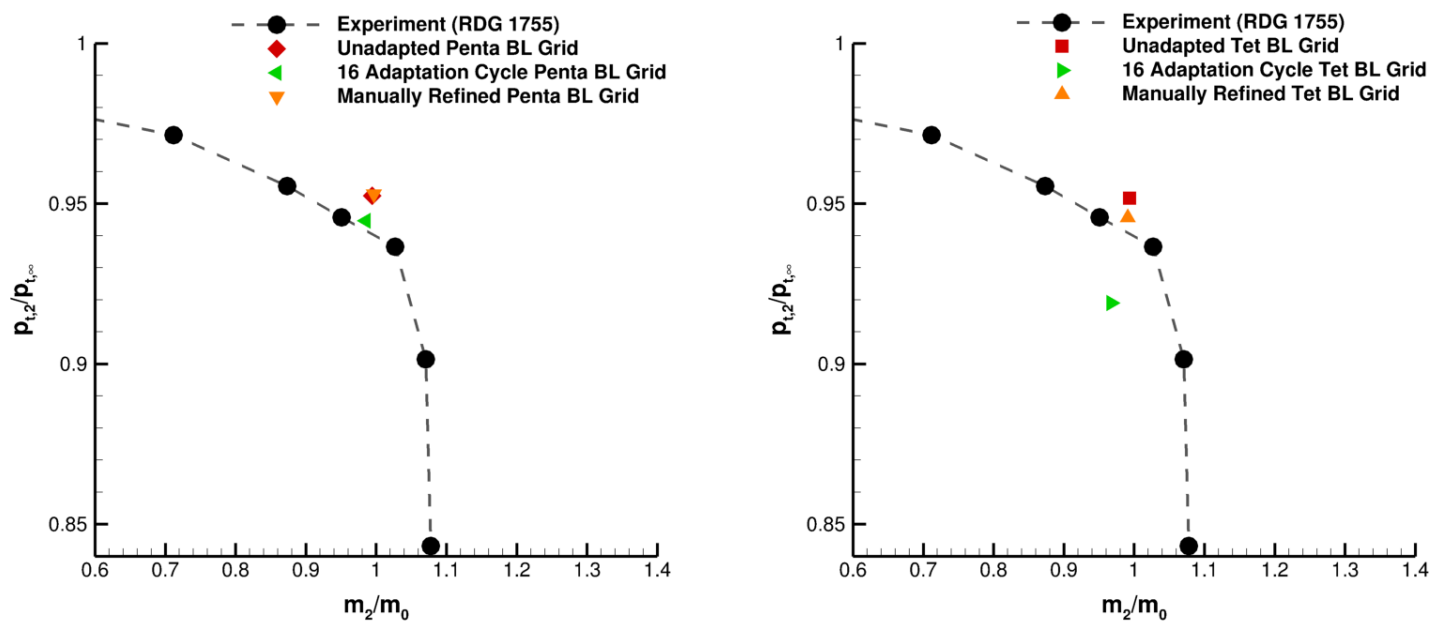

Figure 37.40-probe total pressure recovery plots on the pentahedral boundary-layer grids (left) and on the tetrahedral boundary-layer grids (right) for $M_{\alpha}=1.46, \alpha=2.0^{\circ}$. 

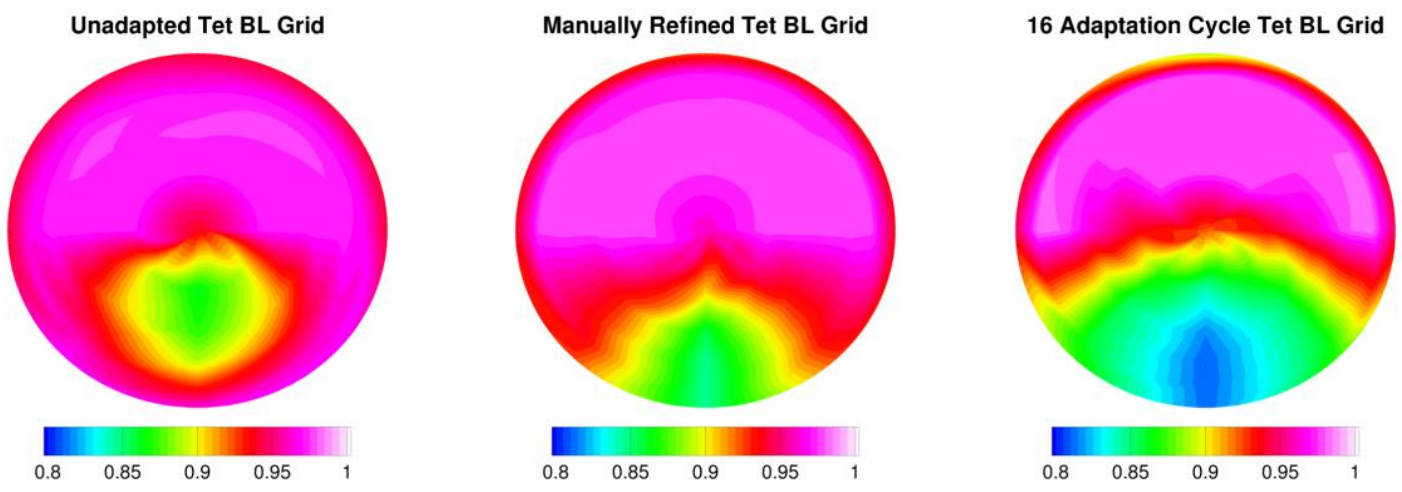

Figure 38.40-probe total pressure recovery contours for the unadapted (left), manually refined(middle), and 16 adaptation cycle (right) tetrahedral boundary-layer grids at $M_{\propto}=1.46, \alpha=2.0^{\circ}$.
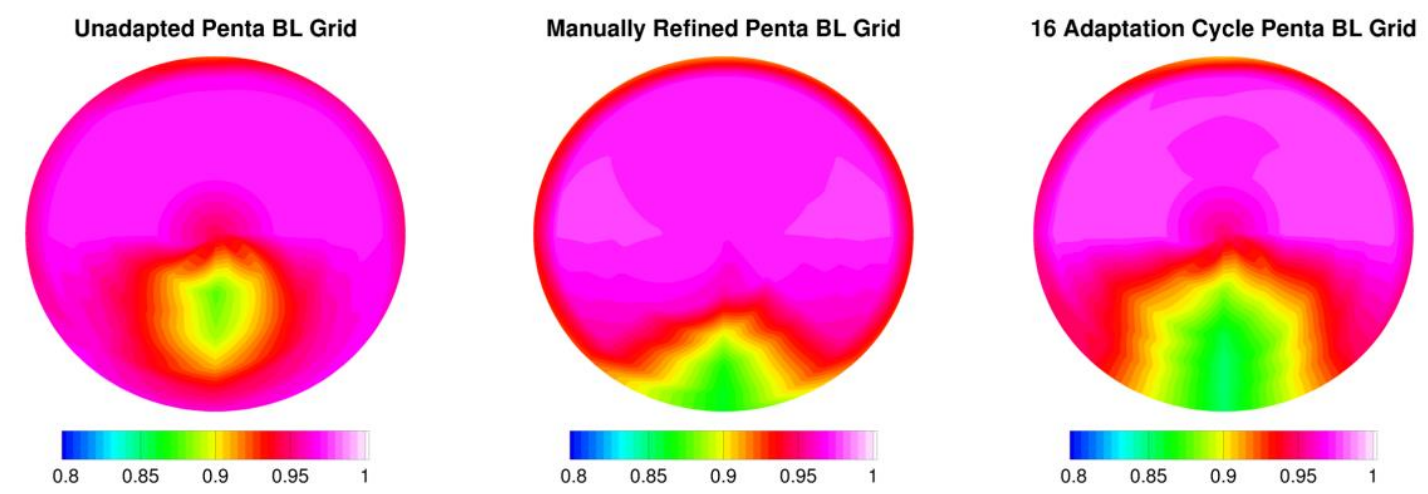

Figure 39.40-probe total pressure recovery contours for the unadapted (left), manually refined(middle), and 16 adaptation cycle (right) pentahedral boundary-layer grids at $M_{\propto}=1.46, \alpha=2.0^{\circ}$.

\section{E. Additional Simulations}

Based on the results obtained on the adapted and manually refined grids, it was decided to run the simulations at the other two conditions using only the unad apted tetrahedral and pentahedral boundary-layergrids. Figure 40 and Fig. 41 show the aircraft centerline Mach number contours at the Reading 1771 conditions $\left(M_{\infty}=1.35, \alpha=3.0^{\circ}\right)$ with an inlet mas s flow rate ratio of 0.96 . Both grids predicta small separation within the subsonic diffuser, a trend that is consistent with the sub-studies using these grids at the higher Mach number condition. Fig ure 42 and Fig. 43 show the static pressure profiles at the camera fairing and inlet bump, respectively, for the CFD solutions on the unad apted tetrahedral boundary-layer grid and the experiment at various inlet mass flow rate ratios at the Re ading 1771 condition. The figures show that the pressure profiles are insensitive to theinlet mass flow rate ratio at the camera fairing region and for the first five upstream pressure stations of the inlet bump region. However, the pres sure measurements at the remaining three downstreamstations are shown to decrease as the mass flow rate ratio is increased. This is because the shockwave in front of the inlet moves downstreamas the inlet mass flow rate ratio is increased. Note that the CFD solutions on the unadapted pentahedral boundary -layer grid were omitted fromFig. 42 and Fig. 43 as they dis played the same trend as the CFD solutions on the unadapted tetrahedral bo und ary -la yer grid. To further compare theCFD solutions and theexperimental data, the static pressure profiles at the camera fairing and inlet bump at an inlet mass flow rate ratio of 0.92 are shown in Fig. 44. While from an engineering pers pective the pressure profiles at the camera faring and at the inlet bump all agree with each o ther, statis tically they do not. Most of the pressure profiles do not agree statistically at the $95 \%$ confidence level because two times the standard deviations between the average pressure meas urements across the various inlet mass flow rate ratios are smaller than the difference between the average pressure measurements. The exception to this is when comparing the CFD solutions to each other at $x=79.0$ " and downstream of $x=79.6$ ". In addition, the 40 -probe av erage total pressure recovery values, shown in Fig. 45, tend to agree statistically with the experimental data for both the unadapted tetrahedral and pentahedral boundary-layer grids at the $95 \%$ confidence level. This is further 
demonstrated in the 40-probe total pressure recovery contour plots, shown in Fig. 46, although both CFD solu tions tend to under predict the size of the lower total pressure recovery region.

Figure 47 and Fig. 48 show the aircraft centerline Mach number contours at the Reading 2033 conditions $\left(M_{\infty}=0.30, \alpha=3.0^{\circ}\right)$ at an inlet mass flow rate ratio of 1.81 for the unadapted tetrahedral boundary-layer grid and 1.82 for the unadapted pentahedreal boundary-layer grid. Unlike the supersonic cases, the small separation reg ion has moved from the bottom of the subsonic diffu ser to the top. This is consistent between the two grids. Figure 49 an $d$ Fig. 50 show the static pressure profiles at the camera fairing and inlet bump, respectively, for the CFD solutions on the unadapted tetrahedral boundary-layer grid and the experiment at various inlet mass flow rate ratios at the Reading 2033 condition. It can be seen that the pressure profiles at the camera fairing are insensitive to the in let mass flow rate ratios with the exception of the CFD solution at $x=44.2 \%$. It can also be shown that the firs three upstream inlet bump stations are insensitive to the inlet mass flow rate ratio while the pressure measurements decrease with increasing mas s flow rate ratio at the remaining stations. This trend is similar to what was seen at the Reading 1771 condition. However unlike the Reading 1771 condition which was at a supersonic freestre am, the decrease in the pressure measurements as the mass flow rate ratio increases is not due to the movement of the external shockwave. To further compare the CFD solutions and the experimental data, the static pressure profiles at the camera fairing and the inlet bump at an inlet mass flow rate ratio of 1.86 are shown in Fig. 51. While from an engineering perspective the static pressure profiles agree well with each other, they in fact do notstatistically ag ree with each other at the $95 \%$ confidence level. There are a few exceptions to this, specifically the CFD solutions compared to the experiment at the most downstreamcamera fairing location, the CFD solutions compared to each other at $x=79.0$ ", $x=79.6$ ", and $x=81.3$ " at the inlet bump, the CFD solution on the tetrahedral boundary-lay er g rid compared to the experiment at $x=81.3$ ", and all data at the most downstreaminlet bump station. Fig u re 52 s hows the respective 40-probe average total pressure recovery values, which show that the CFD solu tion s tend to ag ree well with each other and the experimental data. Statis tically, this is also true at the $95 \%$ confidencelevel with the exception of comparing the average total pressure recoveries for the unadapted te trahed ral boundary-lay er and unadapted pentahedral boundary-layer grids. Differences can also be seen in the 40-probe total pres sure rec ov ery contour plots, shown in Fig. 53. In particular, the CFD solutions tend to not fully capture the lower to tal pressu re recovery region at the lower portion of AIP. Despite the statistical differences, the results of this sub-study sugge st that FUN3D is able to better predict, froman engineering perspective, the flow field and inlet performance of a to paft-mounted propulsion system as the freestreamMach number is decreased.

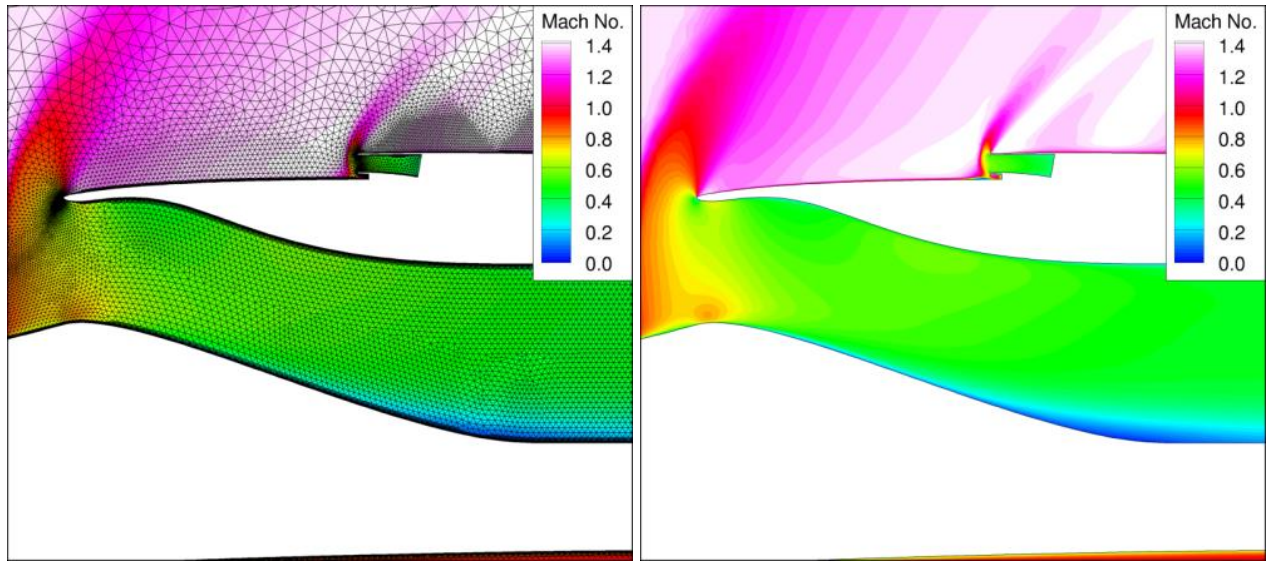

Figure 40. Mach number contours for the unadapted tetrahedral boundary-layer gridfor $M_{\infty}=1.35$, $\alpha=3.0^{\circ}, m_{2} / m_{0}=0.96$. 


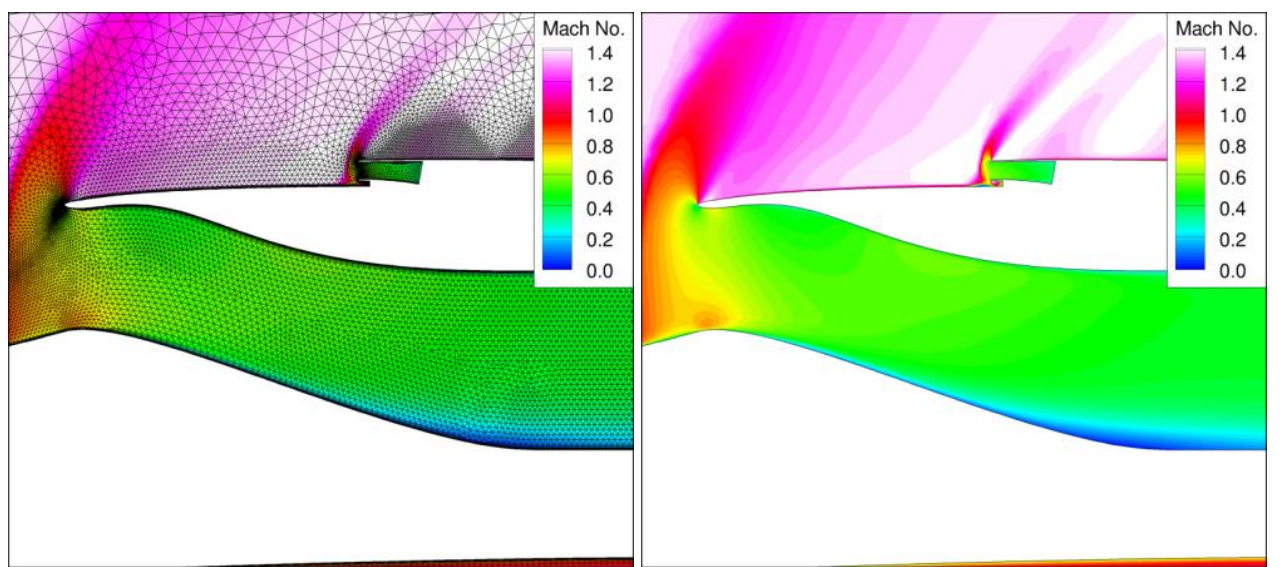

Figure 41. Mach number contours for the unadapted pentahedral boundary-layer grid for $M_{\propto}=1.35$, $\alpha=3.0^{\circ}, m_{2} / m_{0}=0.96$.
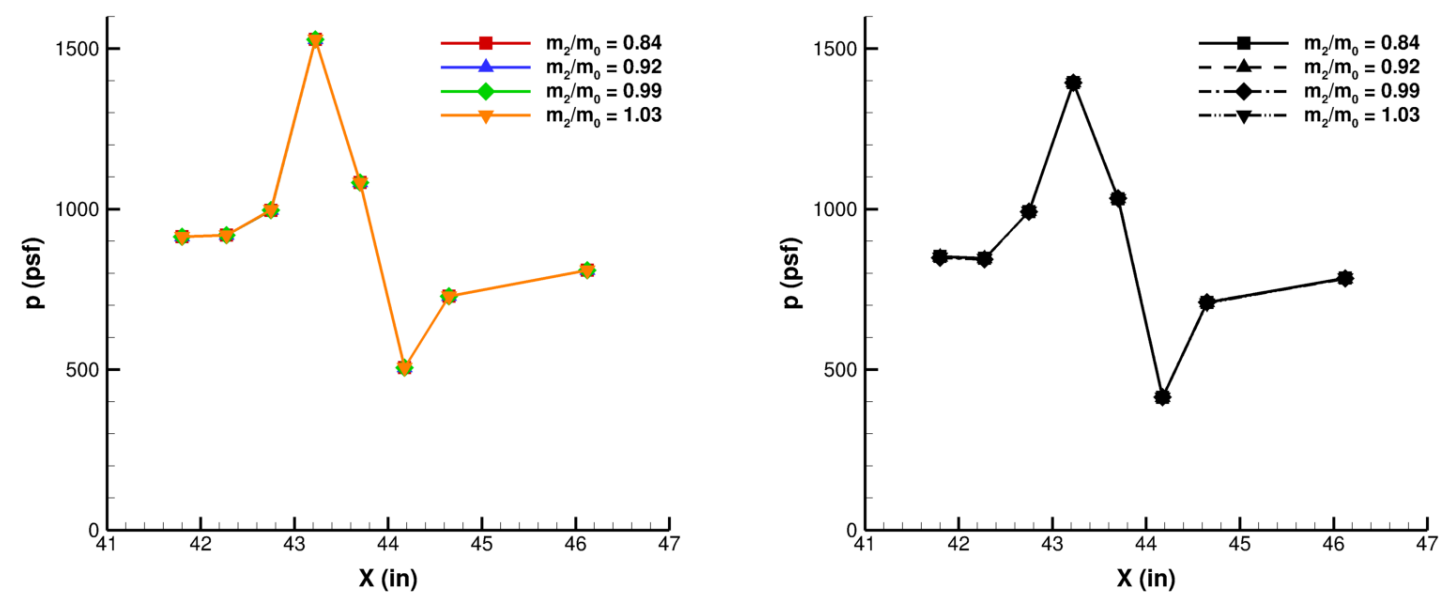

Figure 42. Static pressure profiles along the camera fairing for the unadapted tetrahedral boundary-layer $\operatorname{grid}\left(\right.$ left) and the experiment (right) for $M_{\infty}=1.35, \alpha=3.0^{\circ} . *$
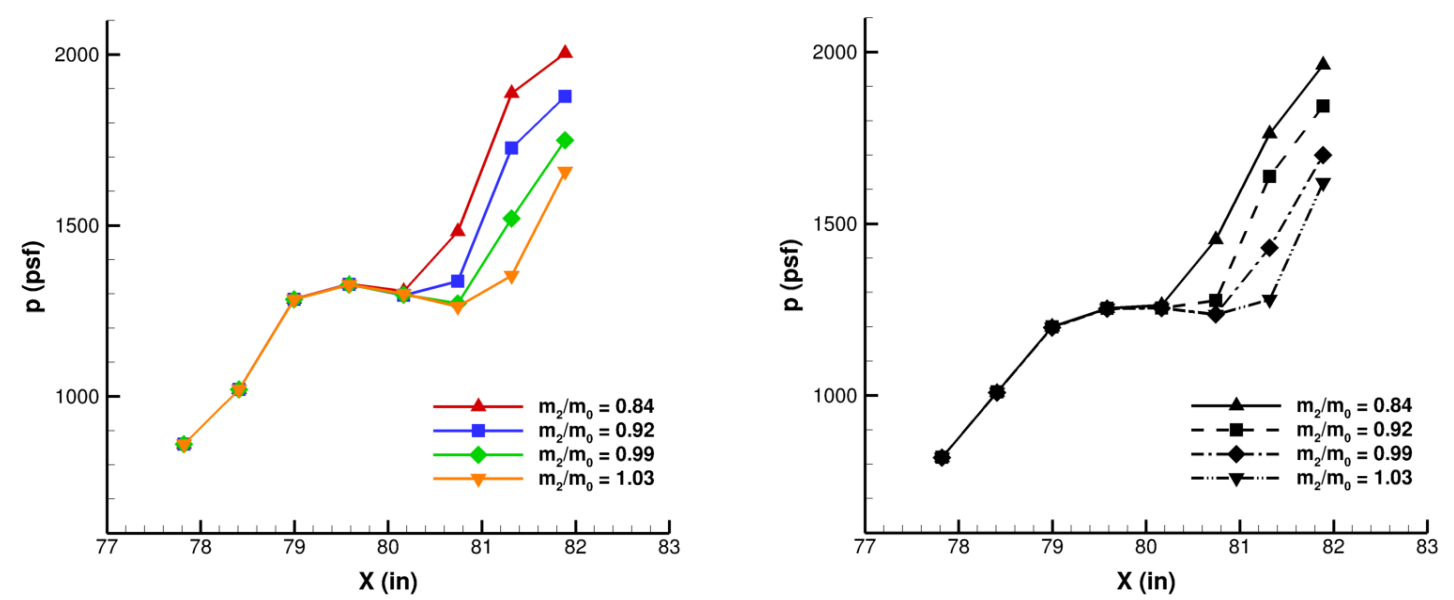

Figure 43. Static pressure profiles along the inlet bump for the unadapted tetrahedral boundary-layer $\operatorname{grid}\left(\right.$ left) and the experiment (right) for $M_{\infty}=1.35, \alpha=3.0^{\circ} . *$ 

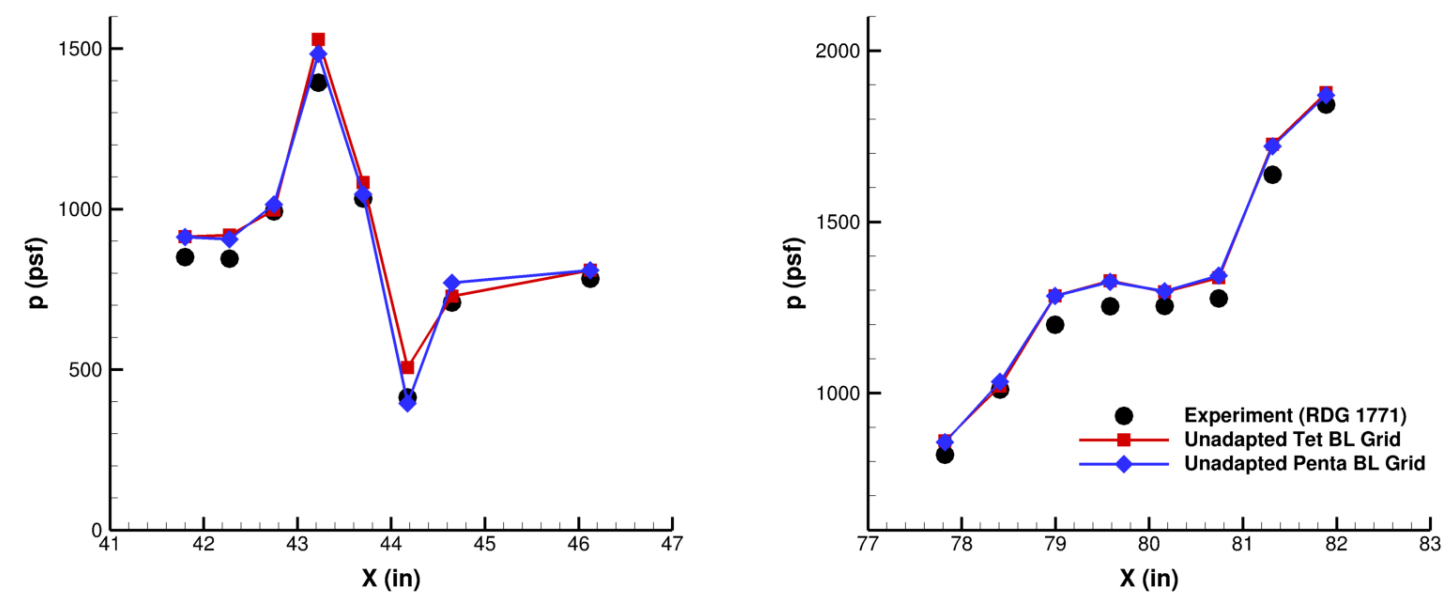

Figure 44. Static press ure profiles along the camera fairing (left) and inlet bump (right) for $M_{\propto}=1.35$, $\alpha=3.0^{\circ}, m_{2} / m_{0}=0.92$. $*$

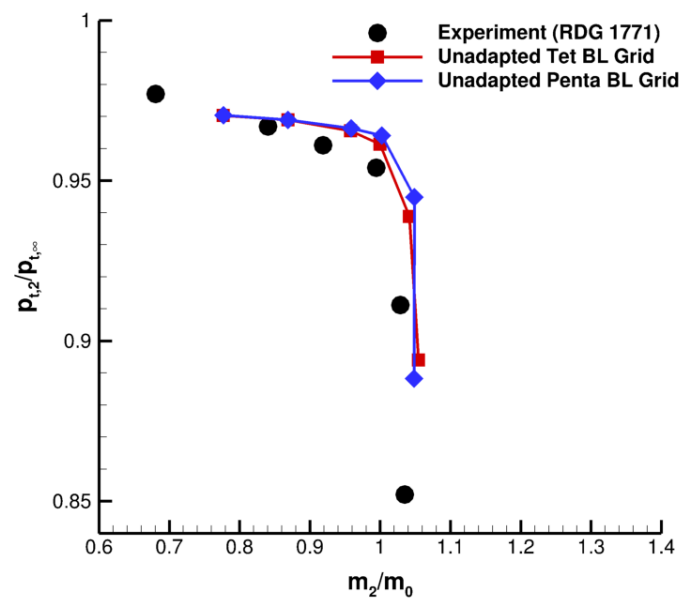

Figure 45.40-probe total pressure recovery plot for $M_{\infty}=1.35, \alpha=3.0^{\circ}$.
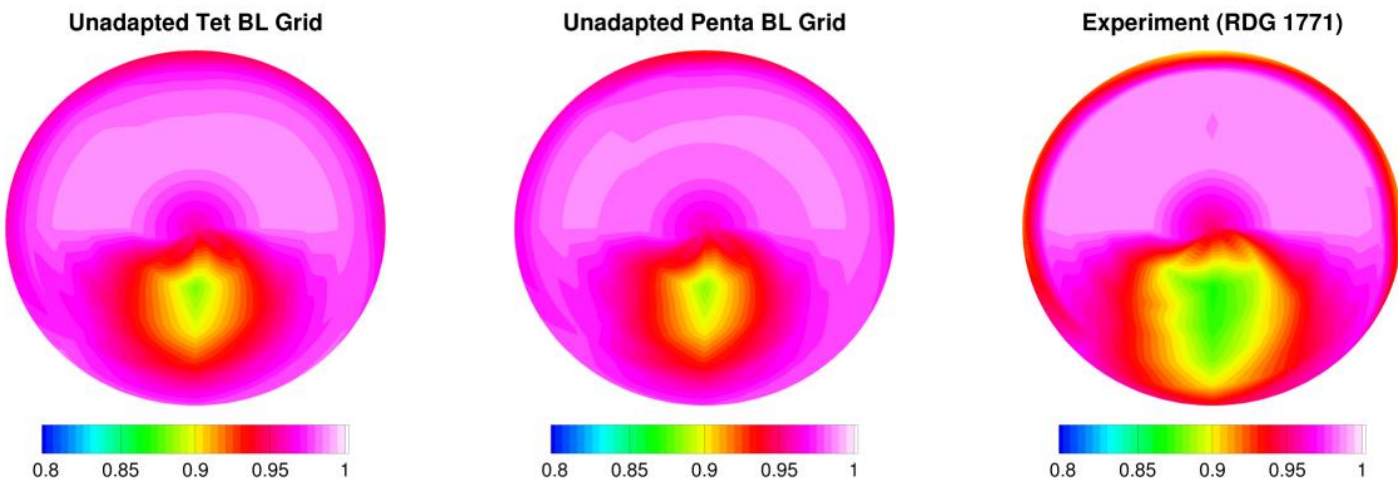

Figure 46.40-probe total pressure recovery contours for the unadapted tetrahedral boundary-layer (left) and the unadapted pentahedral boundary-layer (middle) grids compared to the experiment (right) at $M_{\propto}=1.35, \alpha=3.0^{\circ}$. 

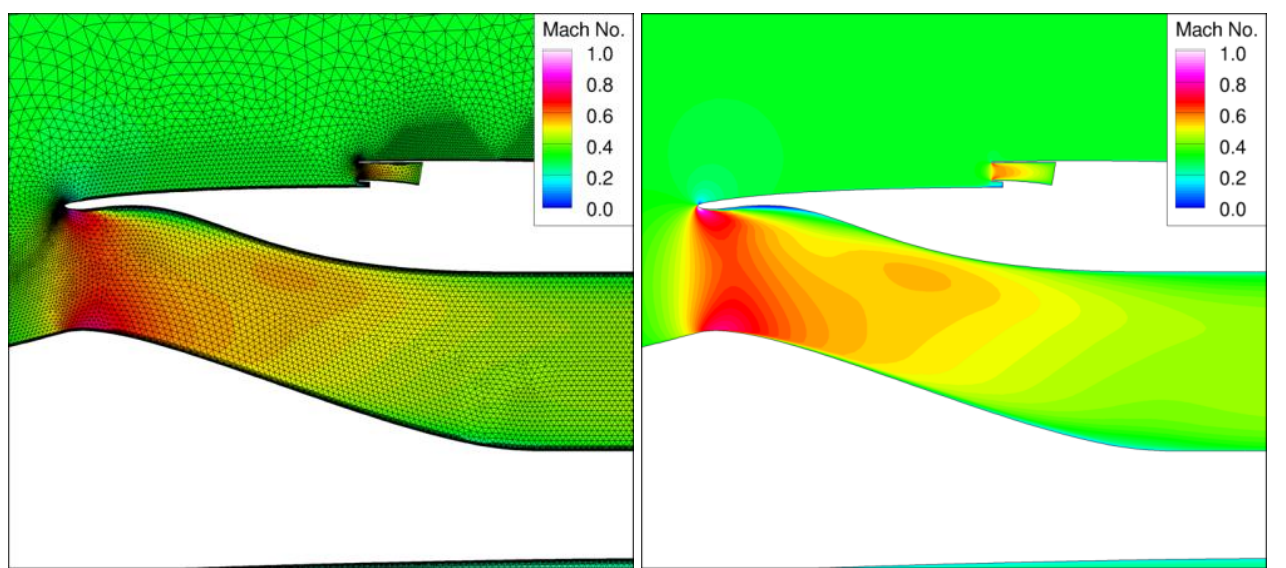

Figure 47. Mach number contours for the unadapted tetrahedral boundary-layer gridfor $M_{\propto}=0.30$, $\alpha=3.0^{\circ}, m_{2} / m_{0}=1.81$.
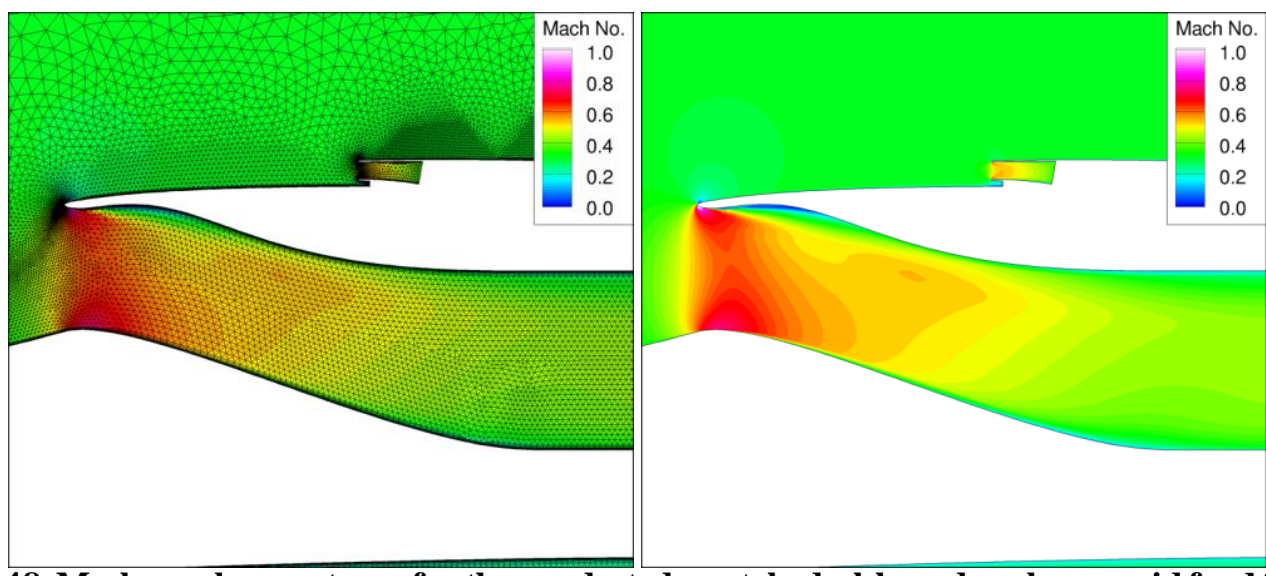

Figure 48. Mach number contours for the unadapted pentahedral boundary-layer grid for $M_{\propto}=0.30$, $\alpha=3.0^{\circ}, m_{2} / m_{0}=1.82$.
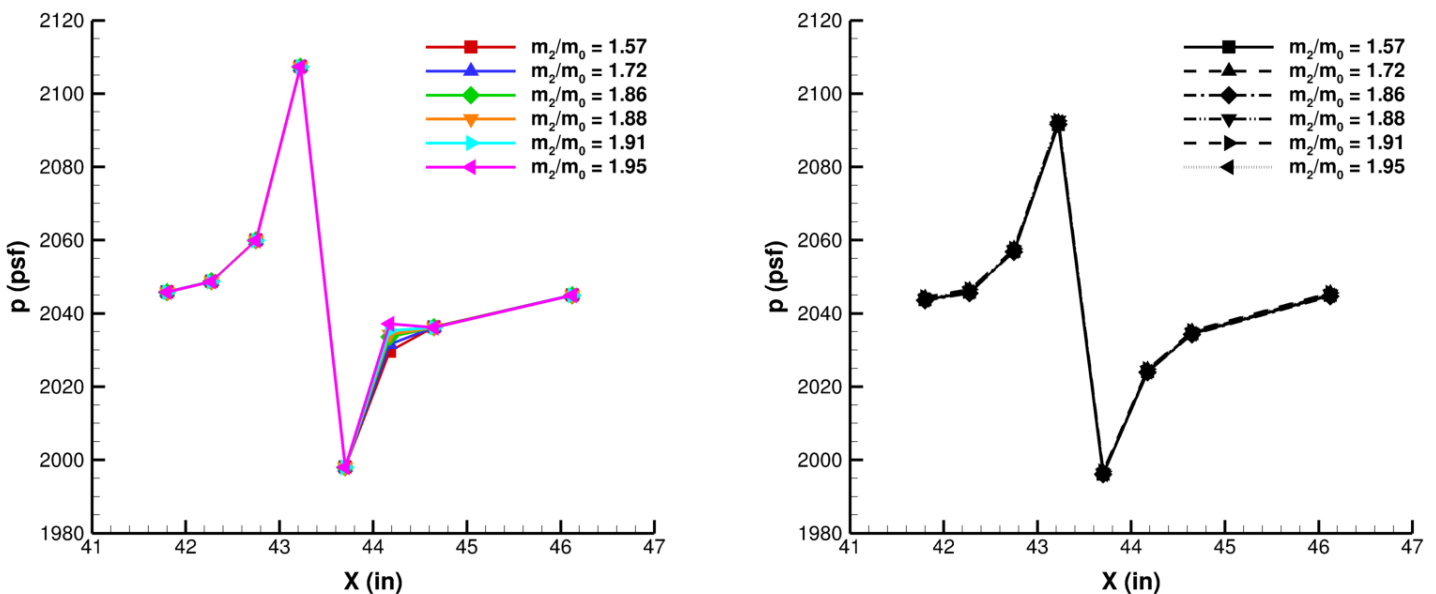

Figure 49. Static pressure profiles along the camera fairing for the unadapted tetrahedral boundary-layer grid (left) and the experiment (right) for $M_{\infty}=0.30, \alpha=3.0 . *$ 

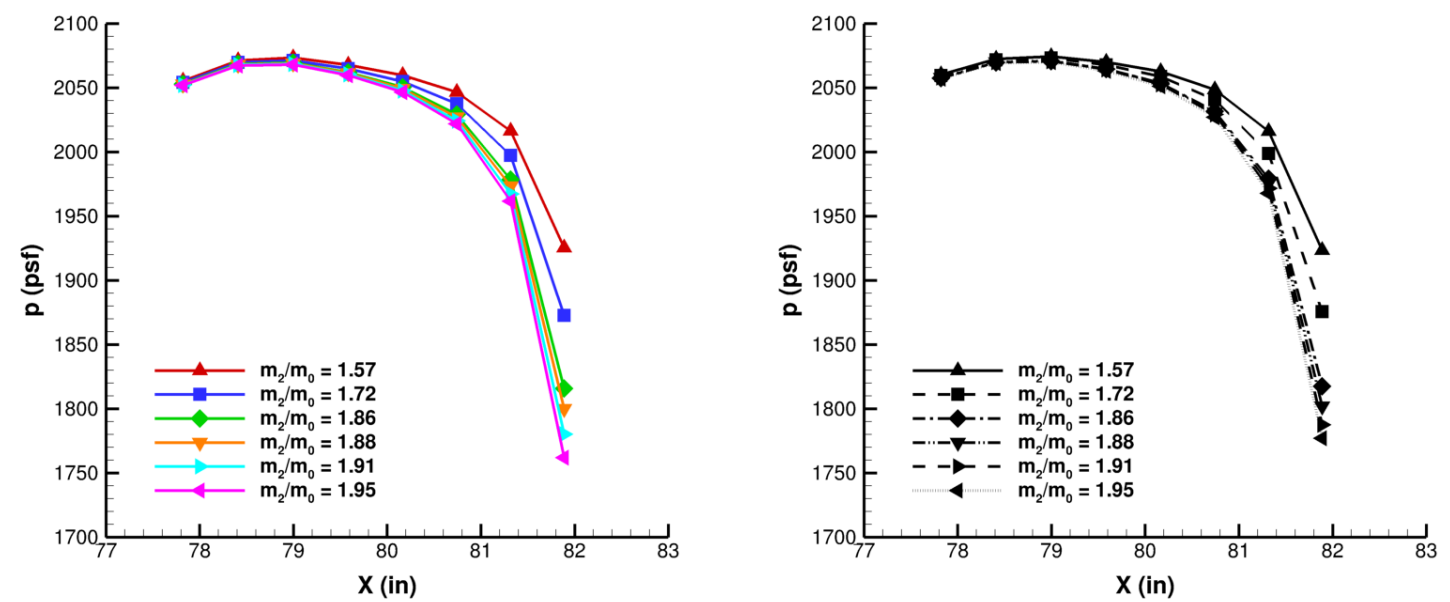

Figure 50. Static pressure profiles along the inlet bump for the unadapted tetrahedral boundary-layer grid(left) and the experiment (right) for $M_{\alpha}=0.30, \alpha=3.0^{\circ} *^{*}$
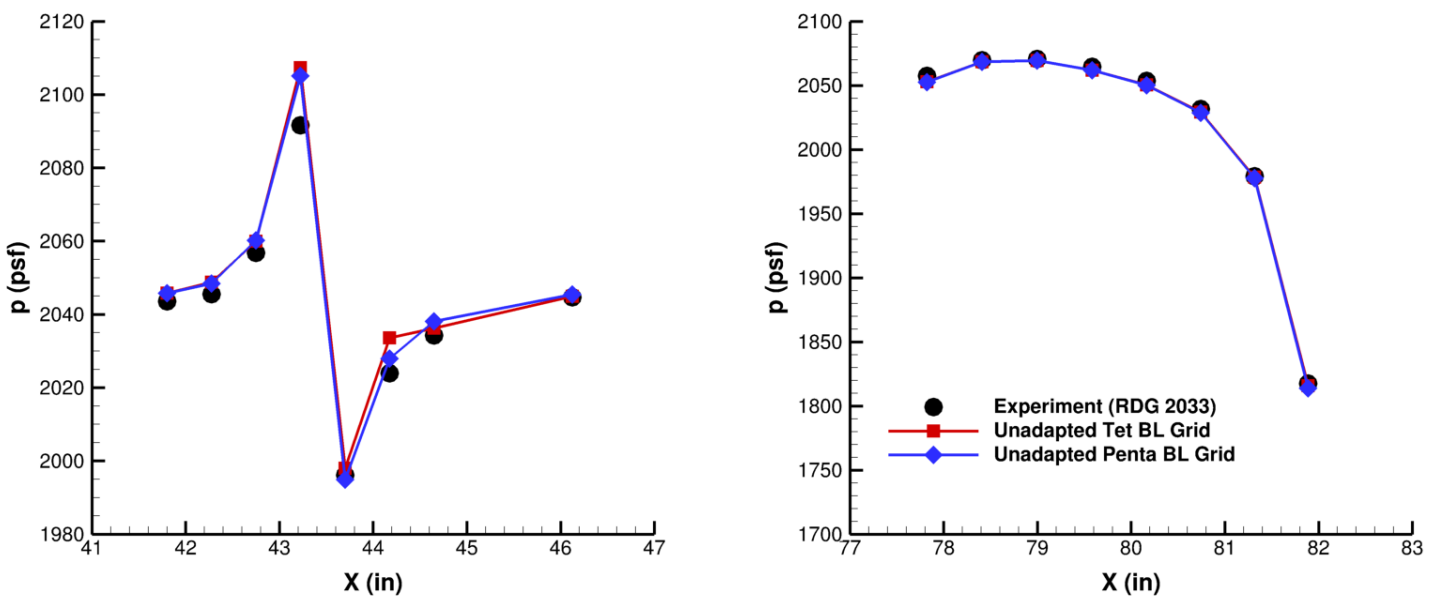

Figure 51. Static press ure profiles al ong the camera fairing (left) and inlet bump (right) for $M_{\propto}=0.30$, $\alpha=3.0^{\circ}, m_{2} / m_{0}=1.86 * *$

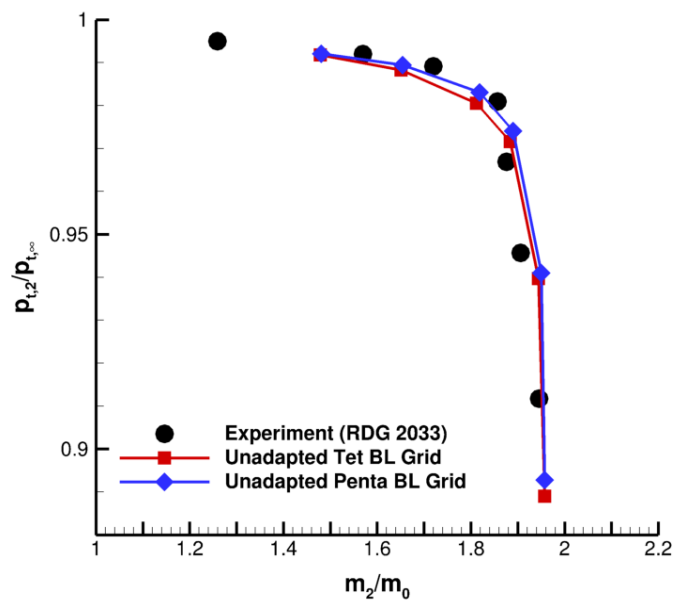

Figure 52.40-probe total pressure recovery plot for $M_{\alpha}=0.30, \alpha=3.0^{\circ}$.

American Institute of Aeronautics and Astronautics 

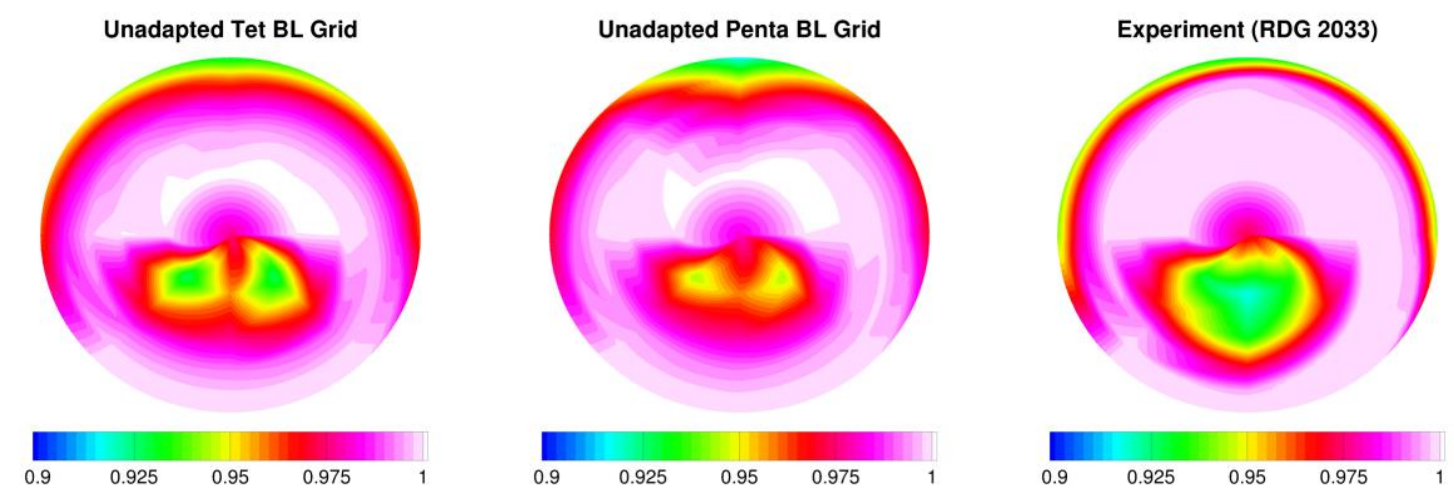

Figure 53.40-probe total pressure recovery contours for the unadapted tetrahedral boundary-layer (left) and the unadapted pentahedral boundary-layer (middle) grids compared to the experiment (right) at

$$
M_{\alpha}=0.30, \alpha=3.0^{\circ} \text {. }
$$

\section{Conclusions}

To conclude, a grid adaptation study was performed on a QueSST aircraft preliminary design in order to determine internal "best practices" for computing inlet performance of top-aft-mountedinlets. It was shown that grids with pentahedral cells in/around the bound ary-layer regions generally did slightly better at pred ic ting in let performance than grids with tetrahedral cells in that same region. It was also shown that both the engine axis aligned linear pressure sensor and the pressure box objective led to adapted grids that poorly predicted inlet performance. In addition, it was shown that the 40-probe total pressure recovery decreases as ymp to tically as the number of adaptation cycles increases and agreement with the experimental data generally got worse with the number of adaptation cycles. Finally, it was shown that the CFD results on the unadapted grids had better agreement with the experimental data at the lower freestream Mach numbers compared to the freestream Mach number of 1.46. These trends suggest that it is hard to predict inlet performance for a high speed top -aft-mo un ted propulsion system without anchoring the CFD solutions to exp erimentaldata and performing a $\mathrm{g}$ rid refinement study.

\section{Appendix A}

The following tables outline the inlet mass flow rate ratios that theCFD simulations were run at.

Table A1: Inlet mass flow rate ratios for the cell type and gridadaptation metric sub-study (tetrahedral boundary-layer grids).

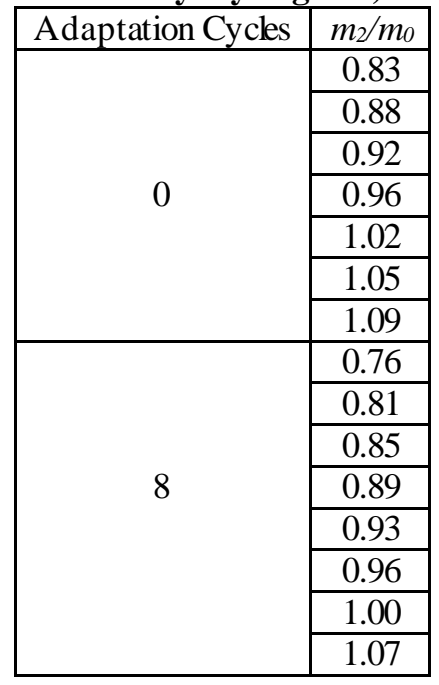

25

American Institute of Aeronautics and Astronautics 
Table A2: Inlet mass flow rate ratios for the cell type and gridadaptation metric sub-study (pentahedral boundary-layer grids).

\begin{tabular}{|c|c|}
\hline Adaptation Cycles & $m_{2} / m_{0}$ \\
\hline \multirow{5}{*}{0} & 0.75 \\
\cline { 2 - 2 } & 0.86 \\
\cline { 2 - 2 } & 0.95 \\
\cline { 2 - 2 } & 1.04 \\
\cline { 2 - 2 } & 1.08 \\
\cline { 2 - 2 } & 1.09 \\
\hline \multirow{5}{*}{8} & 0.75 \\
\cline { 2 - 2 } & 0.80 \\
\hline & 0.85 \\
\cline { 2 - 2 } & 0.89 \\
\cline { 2 - 2 } & 0.94 \\
\cline { 2 - 2 } & 0.99 \\
\cline { 2 - 2 } & 1.03 \\
\cline { 2 - 2 } & 1.07 \\
\cline { 2 - 2 } & 1.10 \\
\hline
\end{tabular}

Table A3: Inlet mass flow rate ratios for the cell type and gridadaptation metric sub-study (pentahedral boundary-layer smooth grid).

\begin{tabular}{|c|c|}
\hline Adaptation Cycles & $m_{2} / m_{0}$ \\
\hline \multirow{4}{*}{0} & 0.86 \\
\cline { 2 - 2 } & 0.95 \\
\cline { 2 - 2 } & 1.04 \\
\cline { 2 - 2 } & 1.08 \\
\cline { 2 - 2 } & 1.09 \\
\hline
\end{tabular}

Table A4: Inlet mass flow rate ratios for the number of adaptation cycles sub-study (tetrahedral boundary-layer grids).

\begin{tabular}{|c|c|}
\hline Adaptation Cycles & $m_{2} / m_{0}$ \\
\hline 0 & 0.99 \\
\hline $8^{*}$ & 0.97 \\
\hline $16^{*}$ & 0.97 \\
\hline
\end{tabular}

*reduced number of additional nodes/adaptation cycle.

Table A5: Inlet mass flow rate ratios for the number of adaptation cycles sub-study (pentahedral boundary-layer grids).

\begin{tabular}{|c|c|}
\hline Adaptation Cycles & $m_{2} / m_{0}$ \\
\hline 0 & 0.99 \\
\hline $8^{*}$ & 0.99 \\
\hline $16^{*}$ & 0.99 \\
\hline
\end{tabular}

*reduced number of additional nodes/adaptation cycle.

Table A6: Inlet mass flow rate ratios for the manually refined grids ub-study (tetrahedral boundary-layer grid).

\begin{tabular}{|c|c|}
\hline Adaptation Cycles & $m_{2} / m_{0}$ \\
\hline 0 & 0.99 \\
\hline
\end{tabular}

Table A7: Inlet mass flow rate ratios for the manually refined gridsub-study (pentahedral boundarylayer grid).

\begin{tabular}{|c|c|}
\hline Adaptation Cycles & $m_{2} / m_{0}$ \\
\hline 0 & 1.00 \\
\hline
\end{tabular}


Table A8: Inlet mass flow rate ratios for the additional simulations sub-study (Reading 1771 conditions, tetrahedral boundary-layer grid).

\begin{tabular}{|c|c|}
\hline Adaptation Cycles & $m_{2} / m_{0}$ \\
\hline \multirow{5}{*}{0} & 0.78 \\
\cline { 2 - 2 } & 0.87 \\
\cline { 2 - 2 } & 0.96 \\
\cline { 2 - 2 } & 1.00 \\
\cline { 2 - 2 } & 1.04 \\
\cline { 2 - 2 } & 1.05 \\
\hline
\end{tabular}

Table A9: Inlet mass flow rate ratios for the additional simulations sub-study (Reading 1771 conditions, pentahedral boundary-layer grid).

\begin{tabular}{|c|c|}
\hline Adaptation Cycles & $m_{2} / m_{0}$ \\
\hline \multirow{4}{*}{0} & 0.78 \\
\cline { 2 - 2 } & 0.87 \\
\cline { 2 - 2 } & 0.96 \\
\cline { 2 - 2 } & 1.00 \\
\cline { 2 - 2 } & 1.05 \\
\cline { 2 - 2 } & 1.05 \\
\hline
\end{tabular}

Table A10: Inlet mass flow rate ratios for the additional simulations sub-study (Reading 2033 conditions, tetrahedral boundary-layer grid).

\begin{tabular}{|c|c|}
\hline Adaptation Cycles & $m_{2} / m_{0}$ \\
\hline \multirow{4}{*}{0} & 1.48 \\
\cline { 2 - 2 } & 1.65 \\
\cline { 2 - 2 } & 1.81 \\
\cline { 2 - 2 } & 1.88 \\
\cline { 2 - 2 } & 1.94 \\
\cline { 2 - 2 } & 1.96 \\
\hline
\end{tabular}

Table A11: Inlet mass flow rate ratios for the additional simulations sub-study (Reading 2033 conditions, pentahedral boundary-layer grid).

\begin{tabular}{|c|c|}
\hline Adaptation Cycles & $m_{2} / m_{0}$ \\
\hline \multirow{4}{*}{0} & 1.48 \\
\cline { 2 - 2 } & 1.65 \\
\cline { 2 - 2 } & 1.82 \\
\cline { 2 - 2 } & 1.89 \\
\cline { 2 - 2 } & 1.95 \\
\cline { 2 - 2 } & 1.96 \\
\hline
\end{tabular}

\section{Appendix B}

The following tables summarize the average and two times the standard deviation values from the paired t-tests.

Table B1: CFD solutions on the unadapted pentahetral boundary-layer grid compared to experimental data at the Reading 1755 condition.

\begin{tabular}{|l|c|c|}
\cline { 2 - 3 } \multicolumn{1}{c|}{} & Average & $2 \sigma$ \\
\hline$p_{t, 2} / p_{t, \infty}$ & 0.010 & 0.014 \\
\hline$D P C P$ & 0.044 & 0.009 \\
\hline$D P R P$ & -0.013 & 0.006 \\
\hline
\end{tabular}


Table B2: CFD solutions on the unadapted tetrahetral boundary-layer gridcompared to experimental data at the Reading 1755 condition.

\begin{tabular}{|l|c|c|}
\cline { 2 - 3 } \multicolumn{1}{c|}{} & Average & $2 \sigma$ \\
\hline$p_{t, 2} / p_{t, \infty}$ & 0.003 & 0.010 \\
\hline$D P C P$ & 0.007 & 0.026 \\
\hline$D P R P$ & -0.015 & 0.008 \\
\hline
\end{tabular}

Table B3: CFD solutions on the unadapted pentahetral boundary-layer smooth grid compared to experimental data at the Reading 1755 condition.

\begin{tabular}{|c|c|c|}
\cline { 2 - 3 } \multicolumn{1}{c|}{} & Average & $2 \sigma$ \\
\hline$p_{t, 2} / p_{t, \infty}$ & 0.008 & 0.014 \\
\hline$D P C P$ & 0.002 & 0.006 \\
\hline$D P R P$ & -0.011 & 0.002 \\
\hline
\end{tabular}

Table B4: CFD solutions on the 8 adaptation cycle pentahetral boundary-layer gridcompared to experimental data at the Reading 1755 condition.

\begin{tabular}{|l|c|c|}
\cline { 2 - 3 } \multicolumn{1}{c|}{} & Average & $2 \sigma$ \\
\hline$p_{t, 2} / p_{t, \infty}$ & 0.003 & 0.025 \\
\hline$D P C P$ & 0.060 & 0.013 \\
\hline$D P R P$ & -0.028 & 0.008 \\
\hline
\end{tabular}

Table B5: CFD solutions on the 8 adaptation cycle tetrahetral boundary-layer grid compared to experimental data at the Reading 1755 condition.

\begin{tabular}{|l|c|c|}
\cline { 2 - 3 } \multicolumn{1}{c|}{} & Average & $2 \sigma$ \\
\hline$p_{t, 2} / p_{t, \infty}$ & -0.034 & 0.033 \\
\hline$D P C P$ & 0.057 & 0.011 \\
\hline$D P R P$ & -0.022 & 0.014 \\
\hline
\end{tabular}

Table B6: CFD solutions on the unadapted tetrahedral boundary-layer gridcompared to CFD solutions on the unadapted pentahetral boundary-layer grid at the Reading 1755 condition.

\begin{tabular}{|l|c|c|}
\cline { 2 - 3 } \multicolumn{1}{c|}{} & Average & $2 \sigma$ \\
\hline$p_{t, 2} / p_{t, \infty}$ & -0.007 & 0.006 \\
\hline$D P C P$ & -0.037 & 0.034 \\
\hline$D P R P$ & -0.002 & 0.003 \\
\hline
\end{tabular}

Table B7: CFD solutions on the unadapted pentahedral boundary-layer smooth grid compared to CFD solutions on the unadapted pentahetral boundary-layer gridat the Reading 1755 condition.

\begin{tabular}{|l|c|c|}
\cline { 2 - 3 } \multicolumn{1}{c|}{} & Average & $2 \sigma$ \\
\hline$p_{t, 2} / p_{t, \infty}$ & -0.002 & 0.007 \\
\hline$D P C P$ & -0.042 & 0.013 \\
\hline$D P R P$ & 0.002 & 0.004 \\
\hline
\end{tabular}

Table B8: CFD solutions on the 8 adaptation cycle pentahedral boundary-layer gridcompared to CFD solutions on the unadapted pentahetral boundary-layer gridat the Reading 1755 condition.

\begin{tabular}{|c|c|c|}
\cline { 2 - 3 } \multicolumn{1}{c|}{} & Average & $2 \sigma$ \\
\hline$p_{t, 2} / p_{t, \infty}$ & -0.007 & 0.014 \\
\hline$D P C P$ & 0.016 & 0.010 \\
\hline$D P R P$ & -0.014 & 0.006 \\
\hline
\end{tabular}


Table B9: CFD solutions on the 8 adaptation cycle tetrahedral boundary-layer grid compared to CFD solutions on the unadapted pentahetral boundary-layer gridat the Reading 1755 condition.

\begin{tabular}{|l|c|c|}
\cline { 2 - 3 } \multicolumn{1}{c|}{} & Average & $2 \sigma$ \\
\hline$p_{t, 2} / p_{t, \infty}$ & -0.044 & 0.028 \\
\hline$D P C P$ & 0.012 & 0.012 \\
\hline$D P R P$ & -0.009 & 0.008 \\
\hline
\end{tabular}

Table B10: CFD solutions on the unadapted pentahedral boundary-layer smooth grid compared to CFD solutions on the unadapted tetrahetral boundary-layer gridat the Reading 1755 condition.

\begin{tabular}{|c|c|c|}
\cline { 2 - 3 } \multicolumn{1}{c|}{} & Average & $2 \sigma$ \\
\hline$p_{t, 2} / p_{t, \infty}$ & 0.005 & 0.007 \\
\hline$D P C P$ & -0.005 & 0.022 \\
\hline$D P R P$ & 0.004 & 0.006 \\
\hline
\end{tabular}

Table B11: CFD solutions on the 8 adaptation cycle pentahedral boundary-layer gridcompared to CFD solutions on the unadapted tetrahetral boundary-layer gridat the Reading 1755 condition.

\begin{tabular}{|l|c|c|}
\cline { 2 - 3 } \multicolumn{1}{c|}{} & Average & $2 \sigma$ \\
\hline$p_{t, 2} / p_{t, \infty}$ & 0.000 & 0.016 \\
\hline$D P C P$ & 0.053 & 0.039 \\
\hline$D P R P$ & -0.012 & 0.006 \\
\hline
\end{tabular}

Table B12: CFD solutions on the 8 adaptation cycle tetrahedral boundary-layer grid compared to CFD solutions on the unadapted tetrahetral boundary-layer gridat the Reading 1755 condition.

\begin{tabular}{|l|c|c|}
\cline { 2 - 3 } \multicolumn{1}{c|}{} & Average & $2 \sigma$ \\
\hline$p_{t, 2} / p_{t, \infty}$ & -0.037 & 0.025 \\
\hline$D P C P$ & 0.050 & 0.035 \\
\hline$D P R P$ & -0.006 & 0.006 \\
\hline
\end{tabular}

Table B13: CFD solutions on the 8 adaptation cycle pentahedral boundary-layer gridcompared to CFD solutions on the unadapted pentahetral boundary-layer smooth gridat the Reading 1755 condition.

\begin{tabular}{|l|c|c|}
\cline { 2 - 3 } \multicolumn{1}{c|}{} & Average & $2 \sigma$ \\
\hline$p_{t, 2} / p_{t, \infty}$ & -0.007 & 0.014 \\
\hline$D P C P$ & 0.016 & 0.010 \\
\hline$D P R P$ & -0.014 & 0.006 \\
\hline
\end{tabular}

Table B14: CFD solutions on the 8 adaptation cycle tetrahedral boundary-layer grid compared to CFD solutions on the unadapted pentahetral boundary-layer smooth gridat the Reading 1755 condition.

\begin{tabular}{|l|c|c|}
\cline { 2 - 3 } \multicolumn{1}{c|}{} & Average & $2 \sigma$ \\
\hline$p_{t, 2} / p_{t, \infty}$ & -0.044 & 0.028 \\
\hline$D P C P$ & 0.012 & 0.012 \\
\hline$D P R P$ & -0.009 & 0.008 \\
\hline
\end{tabular}

Table B15: CFD solutions on the 8 adaptation cycle tetrahedral boundary-layer grid compared to CFD solutions on the 8 adaptation cycle pentahetral boundary-layer gridat the Reading 1755 condition.

\begin{tabular}{|l|c|c|}
\cline { 2 - 3 } \multicolumn{1}{c|}{} & Average & $2 \sigma$ \\
\hline$p_{t, 2} / p_{t, \infty}$ & -0.037 & 0.019 \\
\hline$D P C P$ & -0.003 & 0.006 \\
\hline$D P R P$ & 0.006 & 0.010 \\
\hline
\end{tabular}


Table B16: CFD solutions on the unadapted pentahedral boundary-layer grid compared to experimental data at the Reading 1771 condition (camera fairing pressure measurements).

\begin{tabular}{|c|c|c|}
\cline { 2 - 3 } \multicolumn{1}{c|}{} & Average(psf) & $2 \sigma$ \\
\hline P101 & 62.397 & 2.944 \\
\hline P102 & 61.195 & 2.504 \\
\hline P103 & 21.850 & 1.174 \\
\hline P104 & 90.300 & 0.925 \\
\hline P105 & 13.492 & 1.455 \\
\hline P106 & -18.433 & 1.281 \\
\hline P107 & 61.651 & 2.555 \\
\hline P108 & 25.484 & 1.951 \\
\hline
\end{tabular}

Table B17: CFD solutions on the unadapted pentahedral boundary-layer grid compared to experimental data at the Reading 1771 condition (inlet bump pressure meas urements).

\begin{tabular}{|c|c|c|}
\cline { 2 - 3 } \multicolumn{1}{c|}{} & Average (psf) & $2 \sigma$ \\
\hline P601 & 36.650 & 0.856 \\
\hline P602 & 24.308 & 1.518 \\
\hline P603 & 85.322 & 1.773 \\
\hline P604 & 71.395 & 0.827 \\
\hline P605 & 42.929 & 4.034 \\
\hline P606 & 38.081 & 42.967 \\
\hline P607 & 86.793 & 52.633 \\
\hline P608 & 31.977 & 14.266 \\
\hline
\end{tabular}

Table B18: CFD solutions on the unadapted pentahedral boundary-layer grid compared to experimental data at the Reading 1771 condition (total pressure recovery).

\begin{tabular}{|c|c|c|}
\cline { 2 - 3 } \multicolumn{1}{c|}{} & Average & $2 \sigma$ \\
\hline$p_{t, 2} / p_{t, \infty}$ & 0.009 & 0.017 \\
\hline
\end{tabular}

Table B19: CFD solutions on the unadapted tetrahedral boundary-layer grid compared to experimental data at the Reading 1771 condition (camera fairing press ure measurements).

\begin{tabular}{|c|c|c|}
\cline { 2 - 3 } \multicolumn{1}{c|}{} & Average(psf) & $2 \sigma$ \\
\hline P101 & 63.476 & 2.942 \\
\hline P102 & 73.258 & 2.503 \\
\hline P103 & 4.461 & 1.822 \\
\hline P104 & 135.431 & 0.928 \\
\hline P105 & 49.986 & 1.454 \\
\hline P106 & 92.506 & 1.283 \\
\hline P107 & 19.607 & 2.554 \\
\hline P108 & 24.904 & 1.951 \\
\hline
\end{tabular}

Table B20: CFD solutions on the unadapted tetrahedral boundary-layer grid compared to experimental data at the Reading 1771 condition (inlet bump press ure meas urements).

\begin{tabular}{|l|c|c|}
\cline { 2 - 3 } \multicolumn{1}{c|}{} & Average(psf) & $2 \sigma$ \\
\hline P601 & 40.538 & 0.854 \\
\hline P602 & 11.459 & 1.459 \\
\hline P603 & 85.065 & 1.609 \\
\hline P604 & 74.217 & 0.938 \\
\hline P605 & 43.685 & 3.005 \\
\hline P606 & 38.058 & 29.991 \\
\hline P607 & 94.135 & 42.276 \\
\hline P608 & 40.876 & 12.267 \\
\hline
\end{tabular}


Table B21: CFD solutions on the unadapted tetrahedral boundary-layer grid compared to experimental data at the Reading 1771 condition (total pressure recovery).

\begin{tabular}{|c|c|c|}
\cline { 2 - 3 } \multicolumn{1}{c|}{} & Average & $2 \sigma$ \\
\hline$p_{t, 2} / p_{t, \infty}$ & 0.008 & 0.014 \\
\hline
\end{tabular}

Table B22: CFD solutions on the unadapted pentahedral boundary-layer grid compared to CFD solutions on the unadapted tetrahedral boundary-layer grid at the Reading 1771 condition (camera fairing pressure

\begin{tabular}{|c|c|c|}
\multicolumn{1}{c|}{ meas urements ). } \\
\cline { 2 - 3 } \multicolumn{1}{c|}{} & Average(psf) & $2 \sigma$ \\
\hline P101 & -1.079 & 0.002 \\
\hline P102 & -12.063 & 0.002 \\
\hline P103 & 17.389 & 1.455 \\
\hline P104 & -45.131 & 0.011 \\
\hline P105 & -36.494 & 0.005 \\
\hline P106 & -110.939 & 0.004 \\
\hline P107 & 42.044 & 0.002 \\
\hline P108 & 0.580 & 0.000 \\
\hline
\end{tabular}

Table B23: CFD solutions on the unadapted pentahedral boundary-layer grid compared to CFD solutions on the unadapted tetrahedral boundary-layer grid at the Reading 1771 condition (inlet bump pressure measurements).

\begin{tabular}{|c|c|c|}
\cline { 2 - 3 } \multicolumn{1}{c|}{} & Average (psf) & $2 \sigma$ \\
\hline P601 & -3.887 & 0.014 \\
\hline P602 & 12.849 & 0.115 \\
\hline P604 & 0.257 & 0.377 \\
\hline P605 & -2.823 & 0.493 \\
\hline P606 & -0.756 & 4.900 \\
\hline P607 & -7.342 & 23.223 \\
\hline P608 & -8.899 & 11.984 \\
\hline
\end{tabular}

Table B24: CFD solutions on the unadapted pentahedral boundary-layer grid compared to CFD solutions on the unadapted tetrahedral boundary-layer grid at the Reading 1771 condition (total pressure recovery).

\begin{tabular}{|c|c|c|}
\cline { 2 - 3 } \multicolumn{1}{c|}{} & Average & $2 \sigma$ \\
\hline$p_{t, 2} / p_{t, \infty}$ & 0.001 & 0.005 \\
\hline
\end{tabular}

Table B25: CFD solutions on the unadapted pentahedral boundary-layer grid compared to experimental data at the Reading 2033 condition (camera fairing pressure measurements).

\begin{tabular}{|c|c|c|}
\cline { 2 - 3 } \multicolumn{1}{c|}{} & Average(psf) & $2 \sigma$ \\
\hline P101 & 1.868 & 0.684 \\
\hline P102 & 2.376 & 0.766 \\
\hline P103 & 2.983 & 0.693 \\
\hline P104 & 13.021 & 0.938 \\
\hline P105 & -1.406 & 0.713 \\
\hline P106 & 3.648 & 0.724 \\
\hline P107 & 3.404 & 0.748 \\
\hline P108 & 0.352 & 0.756 \\
\hline
\end{tabular}


Table B26: CFD solutions on the unadapted pentahedral boundary-layer grid compared to experimental data at the Reading 2033 condition (inlet bump press ure measurements).

\begin{tabular}{|l|c|c|}
\cline { 2 - 3 } \multicolumn{1}{c|}{} & Average (psf) & $2 \sigma$ \\
\hline P601 & -5.427 & 0.877 \\
\hline P602 & -1.658 & 0.867 \\
\hline P603 & -1.658 & 0.981 \\
\hline P604 & -3.104 & 1.202 \\
\hline P605 & -4.098 & 1.658 \\
\hline P606 & -3.789 & 2.601 \\
\hline P607 & -2.802 & 4.901 \\
\hline P608 & -5.432 & 12.862 \\
\hline
\end{tabular}

Table B27: CFD solutions on the unadapted pentahedral boundary-layer grid compared to experimental data at the Reading 2033 condition (total pressure recovery).

\begin{tabular}{|l|c|c|}
\cline { 2 - 3 } \multicolumn{1}{c|}{} & Average & $2 \sigma$ \\
\hline$p_{t, 2} / p_{t, \infty}$ & 0.004 & 0.011 \\
\hline
\end{tabular}

Table B28: CFD solutions on the unadapted tetrahedral boundary-layer grid compared to experimental data at the Reading 2033 condition (camera fairing pressure measurements).

\begin{tabular}{|c|c|c|}
\cline { 2 - 3 } \multicolumn{1}{c|}{} & Average(psf) & $2 \sigma$ \\
\hline P101 & 1.876 & 0.682 \\
\hline P102 & 2.702 & 0.765 \\
\hline P103 & 2.718 & 0.691 \\
\hline P104 & 15.220 & 0.943 \\
\hline P105 & 1.664 & 0.721 \\
\hline P106 & 9.247 & 5.353 \\
\hline P107 & 1.508 & 0.745 \\
\hline P108 & -0.175 & 0.754 \\
\hline
\end{tabular}

Table B29: CFD solutions on the unadapted tetrahedral boundary-layer grid compared to experimental data at the Reading 2033 condition (inlet bump press ure meas urements).

\begin{tabular}{|c|c|c|}
\cline { 2 - 3 } \multicolumn{1}{c|}{} & Average(psf) & $2 \sigma$ \\
\hline P601 & -5.120 & 0.859 \\
\hline P602 & -1.775 & 0.843 \\
\hline P603 & -1.674 & 0.945 \\
\hline P604 & -3.075 & 1.159 \\
\hline P605 & -3.965 & 1.607 \\
\hline P606 & -3.579 & 2.493 \\
\hline P607 & -2.364 & 4.685 \\
\hline P608 & -4.514 & 11.936 \\
\hline
\end{tabular}

Table B30: CFD solutions on the unadapted tetrahedral boundary-layer grid compared to experimental data at the Reading 2033 condition (total pressure recovery).

\begin{tabular}{|c|c|c|}
\cline { 2 - 3 } \multicolumn{1}{c|}{} & Average & $2 \sigma$ \\
\hline$p_{t, 2} / p_{t, \infty}$ & 0.002 & 0.011 \\
\hline
\end{tabular}


Table B31: CFD solutions on the unadapted pentahedral boundary-layer grid compared to CFD solutions on the unadapted tetrahedral boundary-layer grid at the Reading 2033 condition (camera fairing press ure measurements).

\begin{tabular}{|c|c|c|}
\cline { 2 - 3 } \multicolumn{1}{c|}{} & Average(psf) & $2 \sigma$ \\
\hline P101 & -0.008 & 0.010 \\
\hline P102 & -0.326 & 0.004 \\
\hline P103 & 0.265 & 0.020 \\
\hline P104 & -2.199 & 0.012 \\
\hline P105 & -3.070 & 0.025 \\
\hline P106 & -5.599 & 5.446 \\
\hline P107 & 1.896 & 0.008 \\
\hline P108 & 0.527 & 0.012 \\
\hline
\end{tabular}

Table B32: CFD solutions on the unadapted pentahedral boundary-layer grid compared to CFD solutions on the unadapted tetrahedral boundary-layer grid at the Reading 2033 condition (inlet bump press ure measurements).

\begin{tabular}{|c|c|c|}
\cline { 2 - 3 } \multicolumn{1}{c|}{} & Average(psf) & $2 \sigma$ \\
\hline P601 & -0.307 & 0.048 \\
\hline P602 & 0.117 & 0.072 \\
\hline P603 & 0.016 & 0.084 \\
\hline P604 & -0.029 & 0.121 \\
\hline P605 & -0.133 & 0.178 \\
\hline P606 & -0.210 & 0.352 \\
\hline P607 & -0.438 & 0.864 \\
\hline P608 & -0.918 & 2.989 \\
\hline
\end{tabular}

Table B33: CFD solutions on the unadapted pentahedral boundary-layer grid compared to CFD solutions on the unadapted tetrahedral boundary-layer grid at the Reading 2033 condition (total pressure recovery).

\begin{tabular}{|l|c|c|}
\cline { 2 - 3 } \multicolumn{1}{c|}{} & Average & $2 \sigma$ \\
\hline$p_{t, 2} / p_{t, \infty}$ & 0.002 & 0.002 \\
\hline
\end{tabular}

\section{Acknowledgments}

The authors would like to thank the NASA Advanced Air Vehicles Program, Commercial Supersonic Technology Project for funding and the NASA's High-End Computing Programfor provid ing s up er-computing resources. The authors would also like to thank Mike Park for guidance on the grid ad aptation process and Stefanie Hirt for providing statistics guidance and the Tecplot macro script used to create the total pres sure recovery cont our plots.

\section{References}

${ }^{1}$ Broge, J. L., "Aerion Progresses on the AS2 with Help from Airbus, and Announces First Fleet Customer," SAE International, URL: http://articles.sae.org/14491/ [cited 30 March 2018].

${ }^{2}$ McMillin, M., "Spike Aerospace Enlarges Supersonic Jet Development Effort," The Weekly Business of Aviation, 26 Jan. 2017 ,pp. 4.

${ }^{3}$ Norris, G., “'Baby Boom' Supersonic Demonstrator Unveiled,” Aviation Daily, 15 Nov. 2016, pp. 3.

${ }^{4}$ Civil Aircraft Sonic Boom, 14 C.F.R. § 91.8172017.

5"NASA Aeronautics Strategic Implementation Plan 2017 Update," NASA Aeronautics Strategic Implementation Plan, URL: https://www.nasa.gov/sites/default/files/atoms/files/sip-2017-03-23-17-high.pdf [cited 1 May 2018].

6"New Aviation Horizons Initiative and Complementary Investments," NASA, URL: https://www.nasa.gov/sites/default/files/atoms/files/nasa-aero-10-yr-plan-508-reduced.pdf [cited 1 May 2018].

${ }^{7}$ Warwick, G., “Affordable Low-Boom X-Plane Goal of Lockheed's QueSST Design," Aviation Week \& Space Technology, 3 March 2016, pp. 21.

${ }^{8}$ Biedron, R. T., et. al., "FUN3D Manual: 13.1,”NASA/TM-2017-219580, February 2017.

${ }^{9}$ Park, M. A., and Carlson, J.-R., “T urbulent Output-Based Anisotropic Adaptation,” AIAA-2010-0168, January 2010. 
${ }^{10}$ Alkandry, H., Park, M. A., Kleb, W. L., and Boyd, I. D., "Feature-Based and Output-Based Grid Adaptation Study for Hypersonic Propulsive Deceleration Jet Flows," Oct ober 2010.

${ }^{11}$ Bartels. R. E., Vatsa, V., Carlson, J.-R., Park, M., and Mineck, R. E., "FUN3D grid Refinement and Adaptation Studies for the Ares Launch Vehicle," AIAA-2010-4372, June 2010.

${ }^{12}$ Park, M. A., and Darmofal, D. L., "Validation of an Output-Adaptive, Tetrahedral Cut-Cell Method for Sonic Boom Prediction," AIAA Journal, Vol. 48, No. 9, 2010, pp. 1928-1945.

${ }^{13}$ Davis, Z. S., and Park, M. A., "IFCPT S-Duct Grid-Adapted FUN3D Computations for the Third Propulsion Aerodynamics Workshop,” AIAA-2017-4835, July 2017.

${ }^{14}$ Point wise, Soft ware Package, Version 17.3 Release 4, Point wise Inc., Fort Worth, TX, 2015.

${ }^{15}$ AFLR3, Software Package, Version 15.5.5, CAVS Sim Center, Mississippi State University, Starkville, MS, 2015.

${ }^{16}$ Marcum, D. L., and Weatherill, N. P., "Unstructured Grid Generation Using Iterative Point Insertion and Local Reconstruction,” AIAA Journal, Vol. 33, No. 9, 1995, pp. 1619-1625.

${ }^{17}$ Marcum, D. L., "Unstructured Grid Generation Using Automatic Point Insertion and Local Reconstruction," The Handbook of Grid Generation, edited by J. F. Thompson, B. Soni, and N. P. Weatherill, CRC Press, 1998, pp. 18-1 through 18-31.

${ }^{18}$ Spalart, P., and Allmaras, S., “A One-Equation Turbulence Model for Aerodynamic Flows," La Recherche Aerospatiale, No. 1, 1984, pp. 5-21.

${ }^{19}$ Society of Automotive Engineers Inc., "Gas T urbine Engine Inlet Flow Distortion Guidelines,” ARP 1420 Rev. B, 2011.

${ }^{20}$ Venditti, D. A., "Grid Adaptation for Functional Outputs of Compressible Flow Simulations," PhD thesis, Massachusetts Institute of T echnology, Cambridge, MA, 2002. 